\title{
Semilinear elliptic equations for the fractional Laplacian with Hardy potential
}

\author{
Mouhamed Moustapha Fall*
}

\begin{abstract}
In this paper we study existence and nonexistence of nonnegative distributional solutions for a class of semilinear fractional elliptic equations involving the Hardy potential.

Key Words: Hardy inequality, critical exponent, nonexistence, distributional solutions, fractional Laplacian.
\end{abstract}

\section{Introduction}

Let $B$ be a ball of $\mathbb{R}^{N}, N>2 s$, centered at 0 . Let $s \in(0,1), p>1$ and $\gamma \geq 0$. In this paper, we study existence and nonexistence of nonnegative functions $u \in \mathcal{L}_{s}^{1} \cap L_{l o c}^{p}(B)$ satisfying

$$
(-\Delta)^{s} u-\gamma|x|^{-2 s} u=u^{p} \quad \text { in } B
$$

where

$$
\mathcal{L}_{s}^{1}=\left\{u: \mathbb{R}^{N} \rightarrow \mathbb{R}: \int_{\mathbb{R}^{N}} \frac{|u|}{1+|x|^{N+2 s}}<\infty\right\}
$$

Equality (0.1) is understood in the sense of distributions. The distribution $(-\Delta)^{s} u$ is defined as

$$
\left\langle(-\Delta)^{s} u, \varphi\right\rangle=\int_{\mathbb{R}^{N}} u(-\Delta)^{s} \varphi d x \quad \forall \varphi \in C_{c}^{\infty}(B) .
$$

*Goethe-Universität Frankfurt, Institut für Mathematik. Robert-Mayer-Str. 10, D-60054 Frankfurt am Main, Germany. E-mail: fall@math.uni-frankfurt.de, mouhamed.m.fall@gmail.com. 
Here the fractional Laplacian $(-\Delta)^{s}$ is defined via the Fourier transform as

$$
(-\Delta)^{s} \varphi(x)=\frac{1}{(2 \pi)^{\frac{N}{2}}} \int_{\mathbb{R}^{N}}|\zeta|^{2 s} \widehat{\varphi}(\zeta) e^{\imath \zeta \cdot x} d \zeta
$$

where

$$
\widehat{\varphi}(\zeta)=\mathcal{F}(\varphi)(\zeta)=\frac{1}{(2 \pi)^{\frac{N}{2}}} \int_{\mathbb{R}^{N}} e^{-\imath \zeta \cdot x} \varphi(x) d x
$$

is the Fourier transform of $\varphi$. The nonlocal structure of the fractional Laplacian $(-\Delta)^{s}$ can be seen in its representation in the real space:

$$
(-\Delta)^{s} \varphi(x)=C_{s, N} P . V . \int_{\mathbb{R}^{N}} \frac{\varphi(x)-\varphi(y)}{|x-y|^{N+2 s}} d y,
$$

for some positive constant $C_{s, N}$. For the equivalence between (0.3) and (0.2), we refer the reader to [33].

Problem (0.1) is related to the relativistic Hardy inequality which were proved by Herbst in [29] (see also [47]):

$$
\gamma_{0} \int_{\mathbb{R}^{N}}|x|^{-2 s} u^{2} d x \leq \int_{\mathbb{R}^{N}}|\zeta|^{2 s} \widehat{u}^{2} d \zeta \quad \forall u \in C_{c}^{\infty}\left(\mathbb{R}^{N}\right),
$$

where

$$
\gamma_{0}=2^{2 s} \frac{\Gamma^{2}\left(\frac{N+2 s}{4}\right)}{\Gamma^{2}\left(\frac{N-2 s}{4}\right)} .
$$

The constant $\gamma_{0}$ is optimal and converges to the classical Hardy constant $\frac{(N-2)^{2}}{4}$ when $s \rightarrow 1$. Here $\Gamma$ is the usual gamma function. We should mention that $(0.4)$ is a particular case of the Stein and Weiss inequality, see [43].

A great deal of work is currently been devoted to the study of the fractional Laplacian as it appears in many fields such as probability theory, physics and mathematical finance. We refer the reader to papers [11], [41], [42], [25], [5] (and the references there in) for a nice expository. A good reference for the potential theory of $(-\Delta)^{s}$ can be found in the book of Landkof [33]. The operator $(-\Delta)^{s}-\gamma_{0}|x|^{-2 s}$ appears in the problem of stability of relativistic matter in magnetic fields. One can see [40] where a lower bound and a Gagliardo-Nirenberg-type inequality were proved. 
The problem of existence and nonexistence of (0.1), for $s=1$, was studied by Brezis-Dupaigne-Tesei in [7] where the authors showed that for $\beta \in\left[0, \frac{N-2}{2}\right], 1<$ $p<\frac{N+2-2 \beta}{N-2-2 \beta}$, the problem

$$
-\Delta u-\left(\frac{(N-2)^{2}}{4}-\beta^{2}\right)|x|^{-2}=u^{p} \quad \text { in } \mathcal{D}^{\prime}(B)
$$

has a positive solution $u \in L^{p}(B)$ and does not have any nonnegative and nontrivial supersolution $u \in L_{l o c}^{p}(B \backslash\{0\})$ when $\beta \in\left[0, \frac{N-2}{2}\right)$ and $p \geq \frac{N+2-2 \beta}{N-2-2 \beta}$. Some related results and problems are in [6], [7], [16], [18], [19], [24], [45], [22], [23], [21], [8], [9].

Our results in this paper extends the one of Brezis-Dupaigne-Tesei in [7] to the case $s \in(0,1)$. Before stating them, we fix the following notation: for $\alpha \in\left[0, \frac{N-2 s}{2}\right)$, we put

$$
\gamma_{\alpha}=2^{2 s} \frac{\Gamma\left(\frac{N+2 s+2 \alpha}{4}\right)}{\Gamma\left(\frac{N-2 s-2 \alpha}{4}\right)} \frac{\Gamma\left(\frac{N+2 s-2 \alpha}{4}\right)}{\Gamma\left(\frac{N-2 s+2 \alpha}{4}\right)} .
$$

The mapping $\alpha \mapsto \gamma_{\alpha}$ is monotone decreasing and $\gamma_{\alpha} \rightarrow 0$ when $\alpha \rightarrow \frac{N-2 s}{2}$. We should mention that this constant appears in the perturbation of the 'ground-state' $|x|^{\frac{2 s-N}{2}}$ for the operator $(-\Delta)^{s}-\gamma_{0}|x|^{-2 s}$. Indeed, letting $\vartheta_{\alpha}=|x|^{\frac{2 s-N}{2}+\alpha}$ we have

$$
(-\Delta)^{s} \vartheta_{\alpha}-\gamma_{\alpha}|x|^{-2 s} \vartheta_{\alpha}=0 \quad \text { in } \mathbb{R}^{N} \backslash\{0\}
$$

see Lemma 3.1 in Section 3.

Our existence result is the following

Theorem 0.1 Let $\alpha \in\left[0, \frac{N-2 s}{2}\right]$ and $1<p<\frac{N+2 s-2 \alpha}{N-2 s-2 \alpha}$. There exits a function $u \in \mathcal{L}_{s}^{1} \cap L^{p}(B)$ satisfying $u>0$ in $B$ and

$$
(-\Delta)^{s} u-\gamma_{\alpha}|x|^{-2 s} u=u^{p} \quad \text { in } \mathcal{D}^{\prime}(B) .
$$

As what concerns nonexistence, we have obtained:

Theorem 0.2 Let $\alpha \in\left[0, \frac{N-2 s}{2}\right)$ and $u \in \mathcal{L}_{s}^{1} \cap L_{l o c}^{p}(B \backslash\{0\})$ such that $u \geq 0$ and

$$
(-\Delta)^{s} u-\gamma_{\alpha}|x|^{-2 s} u \geq u^{p} \quad \text { in } \mathcal{D}^{\prime}(B \backslash\{0\}) .
$$

If $p \geq \frac{N+2 s-2 \alpha}{N-2 s-2 \alpha}$, then $u=0$ in $B$. 
We observe that if $\alpha=0$ we have $p+1=\frac{2 N}{N-2 s}$ : the critical Hardy-LittlewoodSobolev exponent and that $\frac{N+2 s-2 \alpha}{N-2 s-2 \alpha} \rightarrow+\infty$ as $\alpha \rightarrow \frac{N-2 s}{2}$.

The proof of Theorem 0.2 relies on weak comparison principles recently used by the author in [24]. However substantial difficulties have to be overcome due to the nonlocal structure of the fractional Laplacian. Nonexistence result of nonlinear elliptic problems using comparison principles have been obtained in [1], [2] [39], [36], [32], [37], [31] and the references therein.

For the existence result, in the supercritical case $\frac{N+2 s-2 \alpha}{N-2 s-2 \alpha}>p \geq \frac{N+2 s}{N-2 s}$, we have an explicit solution constructed via $\vartheta_{\alpha}$. In the subcritical case, $p+1<\frac{2 N}{N-2 s}$, we used standard variational arguments thanks to the following improved fractional Hardy inequality:

Theorem 0.3 Let $2>q>\max \left(1, \frac{2}{1+2 s}\right)$. Then there exists a constant $C>0$ such that for all $u \in C_{c}^{\infty}(B)$,

$$
C\|u\|_{W_{0}^{\tau, q}(B)}^{2} \leq \gamma_{0} \int_{B}|x|^{-2 s} u^{2} d x-\int_{\mathbb{R}^{N}}|\zeta|^{2 s} \widehat{u}^{2} d \zeta,
$$

where $\tau=\frac{1+2 s}{2}-\frac{1}{q}$.

This result, which might be of self interest, is proved in Appendix 5 .

The proof of all the results presented above are manly based on a Dirichlet-to Neumann operator $\mathcal{B}_{s}$ for which $\mathcal{B}_{s} u=(-\Delta)^{s} \widetilde{u}$ in $\mathcal{D}^{\prime}(E)$ for any Lipschitz bounded open set $E$ of $\mathbb{R}^{N}$ and $\widetilde{u}$ is the null extension outside $E$ of a function $u$ belonging to some Sobolev space. To be more precise let us first recall the result of Caffarelli and Silvestre. We recall that

$$
H^{s}\left(\mathbb{R}^{N}\right)=\left\{u: \mathbb{R}^{N} \rightarrow \mathbb{R}:\left(1+|\zeta|^{s}\right) \widehat{u} \in L^{2}\left(\mathbb{R}^{N}\right)\right\} .
$$

Put

$$
\mathbb{R}_{+}^{N+1}=\left\{(t, x): t>0, x \in \mathbb{R}^{N}\right\} .
$$

Given $w \in H^{s}\left(\mathbb{R}^{N}\right)$, minimization procedure yields the existence of a unique function $\mathcal{H}(w) \in H^{1}\left(\mathbb{R}_{+}^{N+1} ; t^{1-2 s}\right)$ being the harmonic extension of $w$ over the half space $\mathbb{R}_{+}^{N+1}$ :

$$
\left\{\begin{array}{l}
\operatorname{div}\left(t^{1-2 s} \nabla \mathcal{H}(w)\right)=0 \quad \text { in } \mathbb{R}_{+}^{N+1}, \\
\mathcal{H}(w)=w \quad \text { on } \mathbb{R}^{N} .
\end{array}\right.
$$


In [13], Caffarelli and Silvestre proved that $(-\Delta)^{s} w$ is given by the Dirichlet-toNeumann operator $\lim _{t \rightarrow 0} t^{1-2 s} \frac{\partial \mathcal{H}(w)}{\partial t}$ :

$$
-\lim _{t \rightarrow 0} t^{1-2 s} \frac{\partial \mathcal{H}(w)}{\partial t}=\kappa_{s}(-\Delta)^{s} w \quad \text { in } \mathbb{R}^{N}
$$

for some constant $\kappa_{s}>0$. In addition

$$
\int_{\mathbb{R}_{+}^{N+1}} t^{1-2 s}|\nabla \mathcal{H}(w)|^{2} d x d t=\kappa_{s} \int_{\mathbb{R}^{N}}|\zeta|^{2 s} \widehat{w} d \zeta
$$

We want to provide similar arguments in bounded open sets. We define the Hilbert space $\mathscr{D}^{s, 2}\left(\mathbb{R}^{N}\right)$ which is the completion of $C_{c}^{\infty}\left(\mathbb{R}^{N}\right)$ with respect to the norm:

$$
v \mapsto \int_{\mathbb{R}^{N}}|\zeta|^{2 s}|\widehat{v}|^{2} d \zeta
$$

Let $E$ be a bounded open set in $\mathbb{R}^{N}$ with Lipschitz boundary. We introduce the Hilbert space

$$
\mathscr{H}_{0}^{s}(E):=\left\{u \in H^{s}(E): \widetilde{u} \in \mathscr{D}^{s, 2}\left(\mathbb{R}^{N}\right)\right\}
$$

where

$$
\widetilde{u}= \begin{cases}u & \text { in } E \\ 0 & \text { in } \mathbb{R}^{N} \backslash E .\end{cases}
$$

The space $\mathscr{H}_{0}^{s}(E)$ is endowed with the natural norm

$$
\|u\|_{\mathscr{H}_{0}^{s}(E)}^{2}=\int_{\mathbb{R}^{N}}|\zeta|^{2 s}|\widehat{\widetilde{u}}|^{2} d \zeta=\int_{\mathbb{R}^{N}}|\zeta|^{2 s}|\mathcal{F}(\widetilde{u})|^{2} d \zeta
$$

Note that, since $E$ is bounded, by (0.4) there exists a constant $C(E)>0$ such that

$$
C(E)\|\widetilde{u}\|_{H^{s}\left(\mathbb{R}^{N}\right)} \leq\|u\|_{\mathscr{H}_{0}^{s}(E)} \leq\|\widetilde{u}\|_{H^{s}\left(\mathbb{R}^{N}\right)} \quad \forall u \in \mathscr{H}_{0}^{s}(E)
$$

From this we deduce that

$$
\mathscr{H}_{0}^{s}(E)=\left\{u \in H^{s}(E): \widetilde{u} \in H^{s}\left(\mathbb{R}^{N}\right)\right\}
$$

Hence, see for instance [[28], Theorem 1.4.2.2 ], the space $C_{c}^{\infty}(E)$ is dense in $\mathscr{H}_{0}^{s}(E)$. By $(0.11)$ for any $u \in \mathscr{H}_{0}^{s}(E)$, we can consider its harmonic extension $\mathcal{H}(\widetilde{u})$ as in 
(0.7). We define the Dirichlet-to-Neumann operator $\mathcal{B}_{s}: \mathscr{H}_{0}^{s}(E) \rightarrow \mathscr{H}^{-s}(E)$ given by

$$
\mathcal{B}_{s} u=-\kappa_{s}^{-1} \lim _{t \rightarrow 0} t^{1-2 s} \frac{\partial \mathcal{H}(\widetilde{u})}{\partial t},
$$

where $\mathscr{H}^{-s}(E)$ is the dual of $\mathscr{H}_{0}^{s}(E)$. This operator turns out to be linear and it is an isometry,

$$
\left\|\mathcal{B}_{s} u\right\|_{\mathscr{H}^{-s}(E)}=\|u\|_{\mathscr{H}_{0}^{s}(E)} \quad \forall u \in \mathscr{H}_{0}^{s}(E)
$$

by (0.8). Moreover

$$
\mathcal{B}_{s} u=(-\Delta)^{s} \widetilde{u} \quad \text { in } \mathcal{D}^{\prime}(E) \quad \forall u \in \mathscr{H}_{0}^{s}(E) .
$$

In particular a solution $u \in \mathscr{H}_{0}^{s}(E)$ to the problem

$$
\mathcal{B}_{s} u=f \quad \text { in } E
$$

yields a solution $v \in \mathscr{D}^{s, 2}\left(\mathbb{R}^{N}\right)$ to the problem

$$
\left\{\begin{aligned}
(-\Delta)^{s} v=f & \text { in } E, \\
v=0 & \text { in } \mathbb{R}^{N} \backslash E
\end{aligned}\right.
$$

and conversely. We refer to the next section for more details.

In order to get, say, qualitative informations on the solution to the problem

$$
\mathcal{B}_{s} u=(-\Delta)^{s} \widetilde{u}=f,
$$

it is, in general, more convenient to work with the (mixed) problem

$$
\left\{\begin{array}{l}
\operatorname{div}\left(t^{1-2 s} \nabla \mathcal{H}(\widetilde{u})\right)=0 \quad \text { in } \mathbb{R}_{+}^{N+1}, \\
-\lim _{t \rightarrow 0} t^{1-2 s} \frac{\partial \mathcal{H}(\widetilde{u})}{\partial t}=\kappa_{s} f \quad \text { in } E .
\end{array}\right.
$$

New difficulties arise here because of the (possible) degeneracy of the equation (0.12). However the weight $t^{1-2 s}$ falls into the Muckenhoupt class of weights thus regularity results, Harnack inequalities are available (see [20]) and this is enough for our purpose in this paper.

An interesting characterization of $\mathscr{H}_{0}^{s}(E)$, see [28], is that $\mathscr{H}_{0}^{s}(E)$ is the interpolation space $\left(H_{0}^{2}(E), L^{2}(E)\right)_{s, 2}$ :

$$
\mathscr{H}_{0}^{s}(E)=\left\{\begin{array}{c}
H^{s}(E) \quad s \in(0,1 / 2), \\
H_{00}^{s}(E) \quad s=1 / 2, \\
H_{0}^{s}(E) \quad s \in(1 / 2,1),
\end{array}\right.
$$


where

$$
H_{00}^{\frac{1}{2}}(E)=\left\{u \in H^{\frac{1}{2}}(E): \int_{E} \frac{u^{2}(x)}{d(x)} d x<\infty\right\},
$$

endowed with the natural norm, with $d(x)=\operatorname{dist}(x, \partial E)$.

Remark 0.4 Let $E$ be a smooth bounded domain of $\mathbb{R}^{N}$. Recently a pseudo differential operator $A_{s}$ of order $2 s$ was introduced by Cabré and Tan [12] for $s=1 / 2$ (see [15] for every $s \neq 1 / 2$ ) in the following way: for any $u \in \mathscr{H}_{0}^{s}(E)$

$$
A_{s} u=\sum_{k=1}^{\infty} \mu_{k}^{s} u_{k} \varphi_{k}
$$

where $\mu_{k}$ is the zero Dirichlet eigenvalues of $-\Delta$ with corresponding orthonormal eigenfunctions $\varphi_{k}$ and $u_{k}=\int_{E} u \varphi_{k} d x$ is the component of $u$ in the $L^{2}(E)$ basis $\left\{\varphi_{k}\right\}$.

Using (0.13), it was shown in [12] and [15] that

$$
\mathscr{H}_{0}^{s}(E)=\left\{u \in L^{2}(E): \sum_{k=1}^{\infty} \mu_{k}^{s}\left|u_{k}\right|^{2}<\infty\right\} .
$$

The operator $A_{s}$ corresponds to the Dirichlet-to-Neumann operator given by the harmonic extension over the cylinder $E \times(0, \infty)$. Indeed, let $H_{L, s}^{1}(E \times(0, \infty))$ be the set of measurable functions $w: E \times(0, \infty) \rightarrow \mathbb{R}$ with $w \in H^{1}\left(E \times\left(r_{1}, r_{2}\right)\right)$, $0<r_{1}<r_{2}<\infty$ and $w=0$ on $\partial E \times(0, \infty)$ such that the following norm

$$
\|w\|_{H_{L, s}^{1}(E \times(0, \infty))}^{2}=\int_{E \times(0, \infty)} t^{1-2 s}|\nabla w|^{2} d x d t<\infty .
$$

In [12] and [15], the authors showed that for any $g \in \mathscr{H}^{-s}(E)$ there exists a unique solution $u \in \mathscr{H}_{0}^{s}(E)$ to

$$
\left\{\begin{array}{l}
A_{s} u=g \quad \text { in } E \\
u=0 \quad \text { on } \partial E .
\end{array}\right.
$$

In addition $u$ is the trace of $w \in H_{L, s}^{1}(E \times(0, \infty))$ which is the unique solution to

$$
\left\{\begin{array}{l}
\operatorname{div}\left(t^{1-2 s} \nabla w\right)=0 \quad \text { in } E \times(0, \infty), \\
w=0 \quad \text { on } \partial E \times(0, \infty), \\
-t^{1-2 s} \frac{\partial w}{\partial t}=\kappa_{N, s} g \quad \text { on } E
\end{array}\right.
$$


where $\kappa_{N, s}\left(\kappa_{N, s}=1\right.$ for $\left.s=1 / 2\right)$ is a constant depending only on $N$ and $s$. Moreover, it holds that, with the norm in (0.14),

$$
\|u\|^{2}=\kappa_{N, s}\|w\|_{H_{L, s}^{1}(E \times(0, \infty))}^{2} .
$$

We can compare the operator $A_{s}$ with the operator $\mathcal{B}_{s}$. For simplicity, we consider the case $s=1 / 2$. Assume that $g \in C_{c}^{\infty}(E)$ is nonegative and nontrivial and $u$ is a solution to (0.15), which is positive on $E$. Take $w$ its extension over the cylinder. Consider $\mathcal{H}(\widetilde{u})$ which is the harmonic extension of $\widetilde{u}$ in $\mathbb{R}_{+}^{N+1}$ given by (0.12). Clearly

$$
\mathcal{H}(\widetilde{u}) \geq \widetilde{w} \quad \text { in } \overline{\mathbb{R}_{+}^{N+1}} .
$$

It follows from Hopf lemma that

$$
-\frac{\partial w}{\partial t}>-\frac{\partial \mathcal{H}(\widetilde{u})}{\partial t} \quad \text { in } E .
$$

Hence

$$
A_{1 / 2} u>\mathcal{B}_{1 / 2} u \quad \text { in } E \text {. }
$$

In particular the operator $A_{s}$ yields (up to a multiplicative constant) subsolution to the fractional Laplacian $(-\Delta)^{s}$. This is the reason why the use of $\mathcal{B}_{s}$ is more convenient in this paper.

We give here the plan of the paper:

- Section 1: Notations and Preliminaries.

- Subsection 1.1: Dirichlet-to-Neumann operator.

- Section 2: Comparison and maximum principles.

- Section 3: Nonexistence of positive supersolutions.

- Section 4: Existence of positive solutions.

- Appendix 5, Subsection 5.1: Remainder term for the fractional Hardy inequality. 


\section{Notations and Preliminaries}

Let $u \in L^{2}\left(\mathbb{R}^{N}\right)$, we will consider its Fourier transform

$$
\widehat{u}(\zeta)=\mathcal{F}(u)(\zeta):=\frac{1}{(2 \pi)^{\frac{N}{2}}} \int_{\mathbb{R}^{N}} e^{-\imath \zeta \cdot x} u(x) d x .
$$

For $s>0$, the Sobolev space $H^{s}\left(\mathbb{R}^{N}\right)$ is defined as

$$
H^{s}\left(\mathbb{R}^{N}\right)=\left\{u \in L^{2}\left(\mathbb{R}^{N}\right):|\zeta|^{s} \widehat{u} \in L^{2}\left(\mathbb{R}^{N}\right)\right\}
$$

with norm

$$
\|u\|_{H^{s}\left(\mathbb{R}^{N}\right)}=\|\widehat{u}\|_{L^{2}\left(\mathbb{R}^{N}\right)}+\left\||\zeta|^{s} \widehat{u}\right\|_{L^{2}\left(\mathbb{R}^{N}\right)} .
$$

We also have by Parseval identity

$$
\|u\|_{H^{s}\left(\mathbb{R}^{N}\right)}^{2}=\|u\|_{L^{2}\left(\mathbb{R}^{N}\right)}^{2}+C_{s, N} \int_{\mathbb{R}^{N}} \int_{\mathbb{R}^{N}} \frac{|u(x)-u(y)|^{2}}{|x-y|^{N+2 s}} d x d y .
$$

Let $E$ be a bounded domain in $\mathbb{R}^{N}$ with Lipschitz boundary. For $q>1$, we introduce the space $W^{s, q}(E)$ defined as the space of measurable functions $u$ such that the following norm is finite

$$
\|u\|_{W^{s, q}(E)}^{q}:=\|u\|_{L^{q}(E)}^{q}+\int_{E} \int_{E} \frac{|u(x)-u(y)|^{q}}{|x-y|^{N+q s}} d x d y .
$$

We define $W_{0}^{s, q}(E)$ to be the closure of $C_{c}^{\infty}(E)$ with respect to the norm $\|\cdot\|_{W^{s, q}(E)}$. As a notation convention, we put $H^{s}(E)=W^{1,2}(E)$ and $H_{0}^{s}(E)=W_{0}^{1,2}(E)$ which are Hilbert spaces.

It is well known that if $u \in H^{1}(E)$ then $\widetilde{u}$, its null extension outside $E$, is in $H^{1}\left(\mathbb{R}^{N}\right)$ and $\|u\|_{H^{1}(E)}=\|u\|_{H^{1}\left(\mathbb{R}^{N}\right)}$. This is not in general true for functions in $H^{s}(E)(s=1 / 2$ for instance). We shall define a space of functions in which we recover this defect by imposing integrability of null extensions.

The Hardy inequality (0.4) suggests the definition of the Hilbert space $\mathscr{D}^{s, 2}\left(\mathbb{R}^{N}\right)$ which is the completion of $C_{c}^{\infty}\left(\mathbb{R}^{N}\right)$ with respect to the norm:

$$
v \mapsto \int_{\mathbb{R}^{N}}|\zeta|^{2 s}|\widehat{v}|^{2} d \zeta
$$


As it will be apparently clear in the remaining of the paper, we introduce the Hilbert space

$$
\mathscr{H}_{0}^{s}(E):=\left\{u \in H^{s}(E): \widetilde{u} \in \mathscr{D}^{s, 2}\left(\mathbb{R}^{N}\right)\right\},
$$

where we put here (and hereafter)

$$
\widetilde{u}= \begin{cases}u & \text { in } E \\ 0 & \text { in } \mathbb{R}^{N} \backslash E .\end{cases}
$$

The space $\mathscr{H}_{0}^{s}(E)$ is endowed with the norm

$$
\|u\|_{\mathscr{H}_{0}^{s}(E)}^{2}=\int_{\mathbb{R}^{N}}|\zeta|^{2 s}|\widehat{\widetilde{u}}|^{2} d \zeta=\int_{\mathbb{R}^{N}}|\zeta|^{2 s}|\mathcal{F}(\widetilde{u})|^{2} d \zeta .
$$

Note that, since $E$ is bounded, by (0.4) there exists a constant $C(E)>0$ such that

$$
C(E)\|\widetilde{u}\|_{H^{s}\left(\mathbb{R}^{N}\right)} \leq\|u\|_{\mathscr{H}_{0}^{s}(E)} \leq\|\widetilde{u}\|_{H^{s}\left(\mathbb{R}^{N}\right)} \quad \forall u \in \mathscr{H}_{0}^{s}(E) .
$$

Therefore

$$
\mathscr{H}_{0}^{s}(E)=\left\{u \in H^{s}(E): \widetilde{u} \in H^{s}\left(\mathbb{R}^{N}\right)\right\} .
$$

See for instance [[28], Theorem 1.4.2.2 ], the space $C_{c}^{\infty}(E)$ is dense in $\mathscr{H}_{0}^{s}(E)$.

Notations : For $G$ an open set of $\mathbb{R}^{N}$, we use the standard notations for weighted Lebesgue spaces: $L^{p}(G ; a(x))=\left\{u: G \rightarrow \mathbb{R}: \int_{G} u^{p} a(x) d x<\infty\right\}$. $B^{N}(0, r)$ is a ball in $\mathbb{R}^{N}$ centered at 0 with radius $r>0$ and $S^{N-1}=\partial B^{N}(0,1) . \mathbb{R}_{+}^{N+1}=\{(t, x)$ : $\left.t>0, x \in \mathbb{R}^{N}\right\} . B_{+}^{N+1}(0, r)=\mathbb{R}_{+}^{N+1} \cap B^{N+1}(0, r)$ and $S_{+}^{N}=\mathbb{R}_{+}^{N+1} \cap S^{N}$. If there is no confusion, we will put $B^{N}=B^{N}(0,1)$ and $B_{+}^{N+1}=B_{+}^{N+1}(0,1)$. The space $W_{0, S}^{1, q}\left(B_{+}^{N+1}(0, r) ; a(x)\right)=\left\{u \in W^{1, q}\left(B_{+}^{N+1}(0, r) ; a(x)\right): u=0\right.$ on $\left.S_{+}^{N}\right\}$.

\section{$1.1 \quad$ Dirichlet-to-Neumann operator}

It is well known that the space of Schwartz functions $\mathcal{S}$ contains $C_{c}^{\infty}\left(\mathbb{R}^{N}\right)$ and that $\mathcal{F}$ is a bijection from $\mathcal{S}$ into itself. In particular $(-\Delta)^{s} \varphi \in C^{\infty}\left(\mathbb{R}^{N}\right) \cap L^{\infty}\left(\mathbb{R}^{N}\right)$ for every $\varphi \in C_{c}^{\infty}\left(\mathbb{R}^{N}\right)$. In fact we have for any $\varphi \in C_{c}^{2}\left(\mathbb{R}^{N}\right)$, see [41],

$$
\left|(-\Delta)^{s} \varphi(x)\right| \leq C \frac{\|\varphi\|_{C_{c}^{2}}}{1+|x|^{N+2 s}} \quad \forall x \in \mathbb{R}^{N} .
$$

This motivates the following: 
Definition 1.1 Let $G$ be an open subset of $\mathbb{R}^{N}$. Given $u \in \mathcal{L}_{s}^{1}$, the distribution $(-\Delta)^{s} u \in \mathcal{D}^{\prime}(G)$ is defined as

$$
\left\langle(-\Delta)^{s} u, \varphi\right\rangle=\int_{\mathbb{R}^{N}} u(-\Delta)^{s} \varphi d x \quad \forall \varphi \in C_{c}^{\infty}(G) .
$$

Some recent results conserning $s$-superhamonic functions in the sense of distributions as above are in [41].

Consider the Poisson kernel of $\mathbb{R}_{+}^{N+1}:=\left\{(t, x): t>0, x \in \mathbb{R}^{N}\right\}$

$$
P(t, x)=p_{N, s} t^{2 s} \frac{1}{\left(|x|^{2}+t^{2}\right)^{(N+2 s) / 2}},
$$

where $p_{N, s}$ is a normalization constant, see [11] for an explicit value. Let $u \in \mathcal{L}_{s}^{1}$, we can define

$$
\bar{u}(t, x)=P(t, \cdot) * u=p_{N, s} t^{2 s} \int_{\mathbb{R}^{N}} \frac{u(y)}{\left(|y-x|^{2}+t^{2}\right)^{\frac{N+2 s}{2}}} d y \quad \forall(t, x) \in \mathbb{R}_{+}^{N+1} .
$$

It turns out that

$$
\operatorname{div}\left(t^{1-2 s} \nabla \bar{u}\right)=0 \quad \mathbb{R}_{+}^{N+1} .
$$

Therefore $\bar{u}$ is smooth in $\mathbb{R}_{+}^{N+1}$. Moreover if $u$ is regular in a neighborhood of some point $x_{0}$ then

$$
\lim _{t \rightarrow 0} \bar{u}\left(t, x_{0}\right) \rightarrow u\left(x_{0}\right)
$$

By an argument of [13], we have that

$$
-\lim _{t \rightarrow 0} t^{1-2 s} \frac{\partial \bar{u}}{\partial t}\left(t, x_{0}\right)=\kappa_{s}(-\Delta)^{s} u\left(x_{0}\right),
$$

where the constant $\kappa_{s}$ is explicitly computed in [11]:

$$
k_{s}=\frac{\Gamma(1-s)}{2^{2 s-1} \Gamma(s)} \text {. }
$$

For any $w \in H^{s}\left(\mathbb{R}^{N}\right)$, we denote by $\mathcal{H}(w)$ its unique harmonic extension over $\mathbb{R}_{+}^{N+1}$. Namely (see for instance [13], [11]) $\mathcal{H}(w) \in H^{1}\left(\mathbb{R}_{+}^{N+1} ; t^{1-2 s}\right)$ and

$$
\left\{\begin{array}{l}
\operatorname{div}\left(t^{1-2 s} \nabla \mathcal{H}(w)\right)=0 \quad \text { in } \mathbb{R}_{+}^{N+1}, \\
\mathcal{H}(w)=w \quad \text { on } \mathbb{R}^{N}, \\
-t^{1-2 s} \frac{\partial \mathcal{H}(w)}{\partial t}=\kappa_{s}(-\Delta)^{s} w \quad \text { on } \mathbb{R}^{N} .
\end{array}\right.
$$


In particular if $w \in C_{c}^{2}\left(\mathbb{R}^{N}\right)$ then $\mathcal{H}(w)=P(t, \cdot) * w$. In addition one can check (see [13] ), using integration by parts and the Parseval identity, that

$$
\int_{\mathbb{R}_{+}^{N+1}} t^{1-2 s}|\nabla \mathcal{H}(w)|^{2} d x d t=\kappa_{s} \int_{\mathbb{R}^{N}}|\zeta|^{2 s} \widehat{w} d \zeta=\kappa_{s} \int_{\mathbb{R}^{N}}\left|(-\Delta)^{s / 2} w\right|^{2} d x
$$

Therefore from the definition of the space $\mathscr{H}_{0}^{s}(E)$, we have

$$
\kappa_{s}\|v\|_{\mathscr{H}_{0}^{s}(E)}^{2}=\int_{\mathbb{R}_{+}^{N+1}} t^{1-2 s}|\nabla \mathcal{H}(\widetilde{v})|^{2} d x d t \quad \forall v \in \mathscr{H}_{0}^{s}(E)
$$

where as usual $\widetilde{v}$ is the null extension of $v$ outside $E$.

We now introduce a Dirichlet-to-Neumann operator $\mathcal{B}_{s}$ defined on $\mathscr{H}_{0}^{s}(E)$.

Proposition 1.2 Let $E$ be a bounded open set with Lipschitz boundary. Denote by $\mathscr{H}^{-s}(E)$ the dual of $\mathscr{H}_{0}^{s}(E)$. Then the mapping $\mathcal{B}_{s}: \mathscr{H}_{0}^{s}(E) \rightarrow \mathscr{H}^{-s}(E)$ given by

$$
\left\langle\mathcal{B}_{s} v, \varphi\right\rangle_{\mathscr{H}^{-s}(E), \mathscr{H}_{0}^{s}(E)}=-\kappa_{s}^{-1} \int_{\mathbb{R}^{N}} \lim _{t \rightarrow 0} t^{1-2 s} \frac{\partial \mathcal{H}(\widetilde{v})}{\partial t} \widetilde{\varphi} d x \quad \forall v, \varphi \in \mathscr{H}_{0}^{s}(E)
$$

is a linear isometry. In addition for any $v \in \mathscr{H}_{0}^{s}(E)$ we have

$$
\mathcal{B}_{s} v=(-\Delta)^{s} \widetilde{v} \quad \text { in } \mathcal{D}^{\prime}(E) .
$$

Proof. By definition for any $v \in \mathscr{H}_{0}^{s}(E), \widetilde{v} \in H^{s}\left(\mathbb{R}^{N}\right)$ thus the operator $\mathcal{B}_{s}$ is well defined and linear. Consider $\mathcal{H}(\widetilde{v})$ which satisfies (1.8). Then integration by parts yields for every $\varphi \in \mathscr{H}_{0}^{s}(E)$

$$
\int_{\mathbb{R}_{+}^{N+1}} t^{1-2 s} \nabla \mathcal{H}(\widetilde{v}) \cdot \nabla \mathcal{H}(\widetilde{\varphi}) d x d t=\int_{\mathbb{R}^{N}} \lim _{t \rightarrow 0} t^{1-2 s} \frac{\partial \mathcal{H}(\widetilde{v})}{\partial t} \widetilde{\varphi} d x .
$$

This, (1.10) and Hölder inequality imply that

$$
\left\langle\mathcal{B}_{s} v, \varphi\right\rangle_{\mathscr{H}^{-s}(E), \mathscr{H}_{0}^{s}(E)} \leq\|v\|_{\mathscr{H}_{0}^{s}(E)}^{2}\|\varphi\|_{\mathscr{H}_{0}^{s}(E)}^{2}
$$

while

$$
\left\langle\mathcal{B}_{s} v, v\right\rangle_{\mathscr{H}^{-s}(E), \mathscr{H}_{0}^{s}(E)}=\|v\|_{\mathscr{H}_{0}^{s}(E)}^{2}
$$

Hence

$$
\left\|\mathcal{B}_{s} v\right\|_{\mathscr{H}^{-s}(E)}=\|v\|_{\mathscr{H}_{0}^{s}(E)} .
$$


On the other hand, for any $\varphi \in C_{c}^{\infty}(E)$, we have by integration by parts

$$
\begin{aligned}
\int_{\mathbb{R}_{+}^{N+1}} t^{1-2 s} \nabla \mathcal{H}(\widetilde{v}) \cdot \nabla \mathcal{H}(\widetilde{\varphi}) d x d t & =\int_{\mathbb{R}^{N}} \lim _{t \rightarrow 0} t^{1-2 s} \frac{\partial \mathcal{H}(\widetilde{v})}{\partial t} \varphi d x \\
& =\int_{\mathbb{R}^{N}} \lim _{t \rightarrow 0} t^{1-2 s} \frac{\partial \mathcal{H}(\varphi)}{\partial t} \widetilde{v} d x \\
& =\kappa_{s} \int_{\mathbb{R}^{N}} \widetilde{v}(-\Delta)^{s} \varphi d x
\end{aligned}
$$

This means that

$$
\mathcal{B}_{s} v=(-\Delta)^{s} \widetilde{v} \quad \text { in } \mathcal{D}^{\prime}(E)
$$

We turn to the characterization of the space $\mathscr{H}_{0}^{s}(E)$. As suggested with the fact that $\mathcal{H}(\widetilde{v}) \in H^{1}\left(\mathbb{R}_{+}^{N+1} ; t^{1-2 s}\right)$ for every $v \in \mathscr{H}_{0}^{s}(E)$, we have the converse:

Proposition 1.3 Let E be a bounded open set with Lipschitz boundary. Define

$$
H_{0, T}^{1}\left(E ; t^{1-2 s}\right)=\left\{w \in H^{1}\left(\mathbb{R}_{+}^{N+1} ; t^{1-2 s}\right):\left.w\right|_{\mathbb{R}^{N}} \equiv 0 \text { on } \mathbb{R}^{N} \backslash E\right\}
$$

and

$$
H_{0, T}^{s}(E)=\left\{u \in \mathcal{D}^{s, 2}\left(\mathbb{R}^{N}\right): u \equiv 0 \text { in } \mathbb{R}^{N} \backslash E\right\} .
$$

We have the following equalities:

$$
\mathscr{H}_{0}^{s}(E)=\left\{\left.u\right|_{E}: u \in H_{0, T}^{s}(E)\right\}=\left\{\left.w\right|_{E}: w \in H_{0, T}^{1}\left(E ; t^{1-2 s}\right)\right\} .
$$

In particular

$$
(-\Delta)^{s} u=\mathcal{B}_{s} \check{u} \quad \text { in } \mathcal{D}^{\prime}(E), \quad \forall u \in H_{0, T}^{s}(E)
$$

where $\check{u}=\left.u\right|_{E}$.

Proof. The first equality in (1.12) is immediate by definition. The second equality is a consequence of the trace embedding theorem. Indeed, take $w \in H_{0, T}^{1}\left(E ; t^{1-2 s}\right)$. Then the null extension of $\left.w\right|_{E}$ outside $E$ is nothing but $w$ which belongs to $H^{s}\left(\mathbb{R}^{N}\right)$ and in addition $\|w\|_{H^{s}(E)} \leq\|w\|_{H^{s}\left(\mathbb{R}^{N}\right)}$. 
Summarizing, we state the following

Proposition 1.4 Pick $g \in \mathscr{H}^{-s}(E)$. Let $v \in \mathscr{H}_{0}^{s}(E)$ (given by the Lax-Miligram theorem) be the unique solution to

$$
\mathcal{B}_{s} v=g \quad \text { in } E .
$$

Let $w \in H^{1}\left(\mathbb{R}_{+}^{N+1} ; t^{1-2 s}\right)$ solve the mixed problem

$$
\left\{\begin{array}{l}
\operatorname{div}\left(t^{1-2 s} \nabla w\right)=0 \quad \text { in } \mathbb{R}_{+}^{N+1}, \\
w=0 \quad \text { on } \mathbb{R}^{N} \backslash E, \\
-t^{1-2 s} \frac{\partial w}{\partial t}=\kappa_{s} g \quad \text { on } E .
\end{array}\right.
$$

Then $v=w$ in $E$; for any $\varphi \in \mathscr{H}_{0}^{s}(E)$

$$
\begin{aligned}
\left\langle\mathcal{B}_{s} v, \varphi\right\rangle_{\mathscr{H}^{-s}(E), \mathscr{H}^{s}(E)} & =\int_{\mathbb{R}^{N}}|\zeta|^{2 s} \mathcal{F}(\widetilde{v}) \mathcal{F}(\widetilde{\varphi}) \\
& =\int_{\mathbb{R}^{N}}(-\Delta)^{s / 2} \widetilde{v}(-\Delta)^{s / 2} \widetilde{\varphi} d x \\
& =\langle g, \varphi\rangle_{\mathscr{H}^{-s}(E), \mathscr{H} s}(E) \\
& =\kappa_{s}^{-1} \int_{\mathbb{R}_{+}^{N+1}} t^{1-2 s} \nabla w \cdot \nabla \mathcal{H}(\widetilde{\varphi}) d x d t \\
& =\kappa_{s}^{-1} \int_{\mathbb{R}_{+}^{N+1}} t^{1-2 s} \nabla \mathcal{H}(\widetilde{v}) \cdot \nabla \mathcal{H}(\widetilde{\varphi}) d x d t
\end{aligned}
$$

and thus

$$
\kappa_{s}\|v\|_{\mathscr{H}_{0}^{s}(E)}^{2}=\int_{\mathbb{R}_{+}^{N+1}} t^{1-2 s}|\nabla w|^{2} d x d t .
$$

We can extend the above in unbounded domains:

Remark 1.5 Here we consider $E$ any open subset of $\mathbb{R}^{N}$ with Lipschitz boundary. Define

$$
\mathscr{H}^{s}(E):=\left\{u \in H^{s}(E): \widetilde{u} \in H^{1}\left(\mathbb{R}^{N}\right)\right\},
$$

where as usual $\widetilde{u}$ stands for the null extension of u outside $E$. We have that $C_{c}^{\infty}(E)$ is dense in $\mathscr{H}^{s}(E)$, see [28].

By similar arguments, we have that the operator

$$
\overline{\mathcal{B}}_{s}(v)=-\kappa_{s}^{-1} t^{1-2 s} \frac{\partial \mathcal{H}(\widetilde{v})}{\partial t}+v
$$


is a linear isometry form $\mathscr{H}^{s}(E) \rightarrow\left(\mathscr{H}^{s}(E)\right)^{\prime}$, where $\left(\mathscr{H}^{s}(E)\right)^{\prime}$ is the dual of $\mathscr{H}^{s}(E)$.

\section{Comparison and maximum principles}

Unless otherwise stated, $E$ is a bounded Lipschitz open set of $\mathbb{R}^{N}$. We have the following technical result which will be useful in the sequel.

Lemma 2.1 Let $E_{n}$ be a sequence of Lipschitz open sets such that $E_{n} \subset \subset E_{n+1}$ and $\cup_{n=1}^{\infty} E_{n}=E$. Let $g_{n} \in L^{2}(E)$ such that $g_{n} \rightarrow g$ in $L^{2}(E)$. Consider $v_{n} \in \mathscr{H}_{0}^{s}\left(E_{n}\right)$ solution to

$$
\mathcal{B}_{s} v_{n}=g_{n} \quad \text { in } E_{n} .
$$

If $v \in \mathscr{H}_{0}^{s}(E)$ is the unique solution to

$$
\mathcal{B}_{s} v=g \quad \text { in } E
$$

then $\widetilde{v_{n}} \rightarrow v$ in $L^{2}(E)$.

Proof. Observe that $\mathcal{H}\left(\widetilde{v_{n}}\right) \in H_{0, T}^{1}\left(E ; t^{1-2 s}\right)$ thus by Proposition $1.3 \widetilde{v_{n}} \in \mathscr{H}_{0}^{s}(E)$. In addition we have by Hardy and Hölder inequality

$$
\left\|\widetilde{v_{n}}\right\|_{\mathscr{H}_{0}^{s}(E)}=\left\|v_{n}\right\|_{\mathscr{H}_{0}^{s}(E)} \leq C(E)\left\|g_{n}\right\|_{L^{2}(E)} .
$$

Therefore $\widetilde{v_{n}}$ is bounded. By assumption it converges weakly to $v$ in $\mathscr{H}_{0}^{s}(E)$ and strongly in $L^{2}(E)$ because $C_{c}^{\infty}(E)$ is dense in $\mathscr{H}_{0}^{s}(E)$.

The following maximum principle can be found in [[15] Lemma 2.4] or in [20].

Lemma 2.2 Let $E$ be a bounded Lipschitz domain of $\mathbb{R}^{N}$. Let $v \in \mathscr{H}_{0}^{s}(E)$, $v \geq 0$ such that

$$
\mathcal{B}_{s} v \geq 0 \quad \text { in } E \text {. }
$$

If $v \neq 0$ then for any compact set $K \subset E$

$$
e s s \inf _{K} v>0 .
$$


Lemma 2.3 Let $g \in L^{2}(E), g \geq 0$ and let $w \in L_{l o c}^{1}\left(\overline{\mathbb{R}_{+}^{N+1}}\right)$, such that

$$
\int_{\mathbb{R}_{+}^{N+1}} t^{1-2 s}|\nabla w|^{2} d x d t<\infty
$$

and

$$
\int_{\mathbb{R}_{+}^{N+1}} t^{1-2 s} \nabla w \cdot \nabla \phi d x d t+c \int_{E} w \varphi d x d t \geq \kappa_{s} \int_{E} g \phi d x
$$

for every nonegative $\phi \in H_{0, T}^{1}\left(E ; t^{1-2 s}\right)$, where $c \in \mathbb{R}_{+}$. Assume that $w \geq 0$ on $\mathbb{R}^{N} \backslash E$. Then $w \geq 0$ in $\overline{\mathbb{R}_{+}^{N+1}}$.

Proof. Test $(2.1)$ with $\max (-w, 0) \in H_{0, T}^{1}\left(E ; t^{1-2 s}\right)$.

Lemma 2.4 Let $c \in \mathbb{R}_{+}$and let $u \in \mathcal{L}_{s}^{1}, u \geq 0$ and $g \in L^{2}(E)$ such that

$$
(-\Delta)^{s} u+c u \geq g \quad \text { in } \mathcal{D}^{\prime}(E) .
$$

Let $v \in \mathscr{H}_{0}^{s}(E)$ solves

$$
\mathcal{B}_{s} v+c v=g \quad \text { in } E .
$$

Then

$$
u \geq v \quad \text { in } E \text {. }
$$

Proof. Recall that (2.2) is equivalent to

$$
\int_{\mathbb{R}^{N}} u(-\Delta)^{s} \varphi d x \geq \int_{E} g \varphi d x-c \int_{E} u \varphi d x \quad \forall \varphi \in C_{c}^{\infty}(E), \varphi \geq 0 .
$$

Denote by $\rho_{n}$ the standard mollifier (which is symmetric: $\rho_{n}(-x)=\rho_{n}(x)$ ) and put $u_{n}=\rho_{n} * u$.

Claim: for any $\varphi \in C_{c}^{\infty}\left(\mathbb{R}^{N}\right)$

$$
\int_{\mathbb{R}^{N}}(-\Delta)^{s} u_{n} \varphi=\int_{\mathbb{R}^{N}} u(-\Delta)^{s}\left(\rho_{n} * \varphi\right) .
$$

It is easy to check using Fubini's theorem and the symmetry of $\rho_{n}$ that

$$
\int_{\mathbb{R}^{N}}(-\Delta)^{s} u_{n} \varphi d x=\int_{\mathbb{R}^{N}} u_{n}(-\Delta)^{s} \varphi d x=\int_{\mathbb{R}^{N}} u \rho_{n} *(-\Delta)^{s} \varphi d x .
$$


Now we notice that, in $\mathbb{R}^{N}$,

$$
\rho_{n} *(-\Delta)^{s} \varphi=\mathcal{F}\left(\mathcal{F}\left(\rho_{n} *(-\Delta)^{s} \varphi\right)\right)=\mathcal{F}\left(|\zeta|^{2 s}\left(\mathcal{F}\left(\rho_{n}\right) \mathcal{F}(\varphi)\right)\right)=(-\Delta)^{s}\left(\rho_{n} * \varphi\right) .
$$

Using this in (2.6), we get (2.5) as claimed.

Let $E_{n}:=\{x \in E: \operatorname{dist}(x, \partial E)>1 / n\}$. We deduce from (2.4) and (2.5) that for all $\varphi \in C_{c}^{\infty}\left(E_{n}\right)$ and $\varphi \geq 0$

$$
\begin{aligned}
\int_{\mathbb{R}^{N}}(-\Delta)^{s} u_{n} \varphi d x=\int_{\mathbb{R}^{N}} u(-\Delta)^{s}\left(\rho_{n} * \varphi\right) d x & \geq \int_{E} g\left(\rho_{n} * \varphi\right) d x-c \int_{E} u\left(\rho_{n} * \varphi\right) d x \\
& =\int_{E}\left(\rho_{n} * g\right) \varphi d x-c \int_{E}\left(\rho_{n} * u\right) \varphi d x .
\end{aligned}
$$

We conclude that

$$
(-\Delta)^{s} u_{n}(x)+c u_{n}(x) \geq \rho_{n} * g(x)=: g_{n}(x) \quad \text { for every } x \in E_{n} .
$$

We let $w_{n}(t, x)=P(t, \cdot) * u_{n}(x)$ be the harmonic extension of $u_{n}$ via the Poisson kernel so that

$$
\left\{\begin{array}{l}
\operatorname{div}\left(t^{1-2 s} \nabla w_{n}\right)=0 \quad \mathbb{R}_{+}^{N+1}, \\
w_{n}=u_{n} \quad \mathbb{R}^{N}
\end{array}\right.
$$

It turns out that

$$
-t^{1-2 s} \frac{\partial w_{n}}{\partial t}+c w_{n}=\kappa_{s}(-\Delta)^{s} u_{n}+c u_{n} \geq \kappa_{s} g_{n} \quad \text { on } E_{n}
$$

and in addition $t^{1-2 s}\left|\nabla w_{n}\right|^{2} \in L_{l o c}^{1}\left(\overline{\mathbb{R}_{+}^{N+1}}\right)$. Let $v_{n} \in \mathscr{H}_{0}^{s}\left(E_{n}\right)$ be the solution to

$$
\mathcal{B}_{s} v_{n}+c v_{n}=g_{n} \quad \text { in } E_{n} .
$$

We take a large $R>0$ so that $B^{N}(0, R)$ contains $E$ and we let $v_{n, R} \in W_{0, S}^{1,2}\left(B_{+}^{N+1}(0, R) ; t^{1-2 s}\right)$ be the unique solution (obtained by minimization) to the problem

$$
\left\{\begin{array}{l}
\operatorname{div}\left(t^{1-2 s} \nabla v_{n, R}\right)=0 \quad B_{+}^{N+1}(0, R), \\
-t^{1-2 s} \frac{\partial v_{n, R}}{\partial t}+c v_{n, R}=\kappa_{s} g_{n} \quad E_{n}, \\
v_{n, R}=0 \quad B^{N}(0, R) \backslash E_{n} .
\end{array}\right.
$$

By extending $v_{n, R}$ to be zero outside $\overline{B_{+}^{N+1}(0, R)}$, it is standard to show that $v_{n, R} \rightarrow$ $v_{n}$ as $R \rightarrow \infty$ in $H^{1}\left(\mathbb{R}_{+}^{N+1} ; t^{1-2 s}\right)$. Since $w_{n} \geq v_{n, R}$ by Lemma 2.3 , it follows that, sending $R \rightarrow \infty, w_{n} \geq v_{n}$ in $\mathbb{R}^{N}$. In particular $u_{n} \geq v_{n}$ in $E_{n}$. By Lemma 2.1, $\widetilde{v_{n}} \rightarrow v$ in $L^{2}(E)$ and the proof is complete. 
We recall the definition of the $s$-capacity of a compact set $A \subset E$ :

$$
C_{s}(A)=\inf _{\phi \in C_{c}^{\infty}(E)}\left\{\|\phi\|_{\mathscr{H}_{0}^{s}(E)}^{2}: \varphi \geq 1 \text { in a neighborhood of } A\right\} .
$$

Note that if $C_{s}(A)=0$ then $|A|=0$ by Poincaré inequality (see (1.4)). We have the following comparison result modulo small sets.

Lemma 2.5 Let $A$ be a compact subset of $E$ with $C_{s}(A)=0$. Let $u \in \mathcal{L}_{s}^{1}, c \in \mathbb{R}_{+}$ and $g \in L^{2}(E)$ such that

$$
(-\Delta)^{s} u+c u \geq g \quad \text { in } \mathcal{D}^{\prime}(E \backslash A)
$$

Let $v \in \mathscr{H}_{0}^{s}(E)$ solve

$$
\mathcal{B}_{s} v+c v=g \quad \text { in } E .
$$

Then $u \geq v$ in $E$.

Proof. Let $A_{\varepsilon}$ be a smooth open $\varepsilon$-neighborhood of $A$ compactly contained in $E$. Define $D_{\varepsilon}=\left\{x \in D: \operatorname{dist}\left(x, \partial\left(D \backslash A_{\varepsilon}\right)\right)>\varepsilon\right\}$. It is clear that

$$
(-\Delta)^{s} u+c u \geq g \quad \text { in } \mathcal{D}^{\prime}\left(D_{\varepsilon}\right) .
$$

Consider $v_{\varepsilon} \in \mathscr{H}_{0}^{s}\left(D_{\varepsilon}\right)$ solving

$$
\mathcal{B}_{s} v_{\varepsilon}+c v_{\varepsilon}=g \quad \text { in } D_{\varepsilon} .
$$

By Lemma 2.4 we have $u \geq v_{\varepsilon}$ in $D_{\varepsilon}$. The same argument as in the proof of Lemma 2.1 yields $\widetilde{v_{\varepsilon}} \in \mathscr{H}_{0}^{s}(E)$ for every $s \in(0,1)$ and it is bounded. Hence it converges weakly to some function $w$ in $\mathscr{H}_{0}^{s}(E)$ and strongly in $L^{2}(E)$. In particular $u \geq w$. Moreover for any $\varphi \in C_{c}^{\infty}(E \backslash A)$, we can choose $\varepsilon>0$ so small that $\operatorname{supp} \varphi$ is contained in $D_{\varepsilon}$ thus taking the limit as $\varepsilon \rightarrow 0$, we get

$$
\langle w, \varphi\rangle_{\mathscr{H}_{0}^{s}(E)}+c \int_{E} w \varphi=\int_{E} g \varphi d x \quad \forall \varphi \in C_{c}^{\infty}(E \backslash A) .
$$

From this equality, to conclude the proof (that is $v=w$ ), it suffices to show that $C_{c}^{\infty}(E \backslash A)$ is dense in $C_{c}^{\infty}(E)$ with the $\mathscr{H}_{0}^{s}(E)$-norm because $w \in \mathscr{H}_{0}^{s}(E)$.

Since $C_{s}(A)=0$, there exists a sequence $\psi_{n} \in C_{c}^{\infty}(E)$ such that $\psi_{n} \geq 1$ in a neighborhood of $A$ and in addition

$$
\left\|\chi_{n}\right\|_{\mathscr{H}_{0}^{s}(E)}^{2} \leq\left\|\psi_{n}\right\|_{\mathscr{H}_{0}^{s}(E)} \rightarrow 0
$$


where $\chi_{n}=\min \left(\psi_{n}, 1\right)$. Now take any $\phi \in C_{c}^{\infty}(E)$ and note that $\left(1-\chi_{n}\right) \phi \in$ $C_{c}^{\infty}(E \backslash A)$ and moreover $\left(1-\chi_{n}\right) \phi \rightarrow \phi$ in $\mathscr{H}_{0}^{s}(E)$ by $(2.12)$. This concludes the proof.

We shall define a new space which is more convenient when dealing with the Hardy potential. Namely, we assume that there exists $b \in L_{l o c}^{1}(E)$ and a constant $C>0$ such that

$$
\|\varphi\|_{\mathscr{H}_{0}^{s}(E)}^{2}-\int_{E} b(x) \varphi^{2} d x \geq C \int_{E} \varphi^{2} d x \quad \forall \varphi \in C_{c}^{\infty}(E) .
$$

Definition 2.6 Let $b \in L_{\text {loc }}^{1}(E)$ so that (2.13) holds . The Hilbert space $\mathscr{H}_{0, b}^{s}(E)$ is the completion of $C_{c}^{\infty}(E)$ with respect to the scalar product

$$
\langle\varphi, \phi\rangle_{\mathscr{H}_{0}^{s}(E)}-\int_{E} b(x) \varphi \phi d x \quad \forall \varphi, \phi \in C_{c}^{\infty}(E) .
$$

Note that the Lax-Miligram theorem implies that for any $f \in L^{2}(E)$, there exits a unique solution to the problem

$$
\left\{\begin{array}{l}
\mathcal{B}_{s} v-b(x) v=f \quad \text { in } E, \\
v \in \mathscr{H}_{0, b}^{s}(E),
\end{array}\right.
$$

in the sense that for all $\phi \in \mathscr{H}_{0, b}^{s}(E)$

$$
\langle v, \phi\rangle_{\mathscr{H}_{0}^{s}(E)}-\int_{E} b(x) v \phi d x=\int_{E} f \phi d x .
$$

Remark 2.7 Let $\varepsilon>0$. Put $d_{\varepsilon}(x)=b(x)(1-\varepsilon)$. Then $\mathscr{H}_{0}^{s}(E)=\mathscr{H}_{0, d_{\varepsilon}}(E)$ by Propositon 1.3. This holds true because if $v \in \mathscr{H}_{0, d_{\varepsilon}}^{s}(E)$ then by (2.13) we have $\widetilde{v} \in H^{s}\left(\mathbb{R}^{N}\right)$. By similar argument $\mathscr{H}_{0}^{s}(E)=\mathscr{H}_{0, b}^{s}(E)$ if $b \in L^{\infty}(E)$.

Lemma 2.8 Let $A$ be a compact subset of $E$ with $C_{s}(A)=0$. Let $b \in L_{l o c}^{1}(E)$ such that (2.13) holds. Suppose that $u \in \mathcal{L}_{s}^{1}$ with $u, b \geq 0$ and $f \in L^{2}(E), f \geq 0$ such that

$$
(-\Delta)^{s} u-b(x) u \geq f \quad \text { in } \mathcal{D}^{\prime}(E \backslash A) .
$$

Let $v \in \mathscr{H}_{0, b}^{s}(E)$ be the unique solution to

$$
\mathcal{B}_{s} v-b(x) v=f \quad \text { in } E .
$$

Then

$$
u \geq v \quad \text { in } E \text {. }
$$


Proof. Step 1: We first prove the result if $b \in L^{\infty}(E)$.

We let $v_{0} \in \mathscr{H}_{0}^{s}(E)$ solving

$$
\mathcal{B}_{s} v_{0}=f \quad \text { in } E .
$$

Then $0 \leq v_{0} \leq u$ in $E$ by Lemma 2.5 and because $f \geq 0$. We define inductively the sequence $v_{n} \in \mathscr{H}_{0}^{s}(E)$ by

$$
\mathcal{B}_{s} v_{1}=b(x) v_{0}+f \quad \text { in } E, \quad \mathcal{B}_{s} v_{n}=b(x) v_{n-1}+f \quad \text { in } E .
$$

Since $b \geq 0$, we have $(-\Delta)^{s} u \geq b(x) v_{0}+f$ in $\mathcal{D}^{\prime}(E \backslash A)$. Thus using once again Lemma 2.5, we obtain $v_{0} \leq v_{1} \leq u$ in $E$. By induction, we have

$$
v_{0} \leq v_{1} \leq \cdots \leq v_{n} \leq u \quad \text { in } E \quad \forall n \in \mathbb{N} \text {. }
$$

Since $v_{n-1} \leq v_{n}$ in $E$, we have

$$
\left\|v_{n}\right\|_{\mathscr{H}_{0}^{s}(E)}^{2}-\int_{E} b(x)\left|v_{n}\right|^{2} \leq \int_{E} f(x) v_{n} d x .
$$

By Hölder inequality and (2.13) (see Remark 2.7) $v_{n}$ is bounded in $\mathscr{H}_{0}^{s}(E)$. We conclude that $v_{n} \rightarrow v$ in $\mathscr{H}_{0}^{s}(E)$ as $n \rightarrow \infty$ which is the unique solution to

$$
\mathcal{B}_{s} v=b(x) v+f \quad \text { in } E .
$$

Since $v_{n} \rightarrow v$ in $L^{2}(E)$, we get $v \leq u$ in $E$.

Step 2: Conclusion of the proof.

We put $b_{k}(x)=\min (b(x), k)$ for every $k \in \mathbb{N}$. We consider $v^{k} \in \mathscr{H}_{0}^{s}(E)$ be the unique solution to

$$
\left\langle v^{k}, \varphi\right\rangle_{\mathscr{O}_{0}^{s}(E)}-\int_{E} \min \{b(x), k\} v^{k} \varphi=\int_{E} f \varphi \quad \forall \varphi \in C_{c}^{\infty}(E) .
$$

Thanks to Step 1, we have $v^{k} \leq u$ in $E$.

Next, we check that such a sequence $v^{k}$, satisfying (2.16), converges to $v$ in $L^{2}(E)$ when $k \rightarrow \infty$. Indeed, we have

$$
\begin{aligned}
\left\|v^{k}\right\|_{\mathscr{H}_{0, b}^{s}(E)}^{2} & \leq\left\|v^{k}\right\|_{\mathscr{H}_{0}^{s}(E)}^{2}-\int_{E} \min \{b(x), k\}\left|v^{k}\right|^{2} d x \\
& =\int_{E} f v^{k} d x \leq C\left\|v^{k}\right\|_{\mathscr{H}_{0, b}^{s}(E)}
\end{aligned}
$$


by Hölder inequality and by (2.13), where the constant $C$ depends on $f$ and $E$ but not on $k$. Therefore the sequence $v^{k}$ is bounded in $\mathscr{H}_{0, b}^{s}(E)$. We conclude that there exists $\widetilde{v} \in \mathscr{H}_{0, b}^{s}(E)$ such that, for a subsequence, $v^{k} \rightarrow \widetilde{v}$ in $\mathscr{H}_{0, b}^{s}(E)$. Now by $(2.16)$, we have

$$
\left\langle v^{k}, \varphi\right\rangle_{\mathscr{H}_{0, b}^{s}(E)}+\int_{E}(b(x)-\min \{b(x), k\}) v^{k} \varphi=\int_{E} f \varphi .
$$

Since for every $k \geq 1$ and any $\varphi \in C_{c}^{\infty}(E)$

$$
\left|(b(x)-\min \{b(x), k\}) v^{k} \varphi\right| \leq(b(x)-\min \{b(x), k\}) u|\varphi| \leq 2 b(x) u|\varphi| \in L^{1}(E),
$$

the dominated convergence theorem implies that

$$
\langle\widetilde{v}, \varphi\rangle_{\mathscr{H}_{0, b}^{s}(E)}=\int_{E} f \varphi \quad \text { for any } \varphi \in C_{c}^{\infty}(E) .
$$

We therefore have that $\widetilde{v}=v$ by uniqueness. By (2.17), we have

$$
\begin{aligned}
\left\|v-v^{k}\right\|_{\mathscr{H}_{0, b}^{s}(E)}^{2} & =\left\|v^{k}\right\|_{\mathscr{H}_{0, b}^{s}(E)}^{2}-\left\langle v, v^{k}\right\rangle_{\mathscr{H}_{0, b}^{s}(E)}+\left\langle v, v-v^{k}\right\rangle_{\mathscr{H}_{0, b}^{s}(E)} \\
& =\left\|v^{k}\right\|_{\mathscr{H}_{0, b}^{s}(E)}^{2}-\int_{E} f v^{k}+\left\langle v, v-v^{k}\right\rangle_{\mathscr{H}_{0, b}^{s}(E)} \\
& \leq\left\|v^{k}\right\|_{\mathscr{H}_{0}^{s}(E)}^{2}-\int_{E} \min \{b(x), k\}\left|v^{k}\right|^{2} d x-\int_{E} f v^{k}+\left\langle v, v-v^{k}\right\rangle_{\mathscr{H}_{0, b}^{s}(E)} \\
& =\left\langle v, v-v^{k}\right\rangle_{\mathscr{H}_{0, b}^{s}(E)} .
\end{aligned}
$$

We thus obtain

$$
C(E) \int_{\Omega}\left|v-v^{k}\right|^{2} d x \leq\left\langle v, v-v^{k}\right\rangle_{\mathscr{H}_{0, b}^{s}(E)} \rightarrow 0
$$

by (2.13). Hence $v^{k} \rightarrow v$ pointwise and thus $v \leq u$ in $\Omega$.

Remark 2.9 The same result as in Lemma 2.8 holds if we assumed the coercivity that there exist constants $C, c>0$ such that for all $\varphi \in C_{c}^{\infty}(E)$

$$
\|\varphi\|_{\mathscr{H}_{0}^{s}(E)}^{2}+c \int_{E} \varphi^{2} d x-\int_{E} b(x) \varphi^{2} d x \geq C \int_{E} \varphi^{2} d x .
$$

We close this section with the following useful lemma and its immediate consequence. Its counterpart, for $s=1$, is in [23]. 
Lemma 2.10 Let $E$ be a bounded Lipschitz domain of $\mathbb{R}^{N}$. Let $A$ be a compact subset of $E$ with $C_{s}(A)=0$. Let $u \in \mathcal{L}_{s}^{1}, b \in L_{\text {loc }}^{1}(E)$ and $u, b>0$. Assume that

$$
(-\Delta)^{s} u \geq b(x) u \quad \text { in } \mathcal{D}^{\prime}(E \backslash A) .
$$

Then

$$
\int_{\mathbb{R}_{+}^{N+1}} t^{1-2 s}|\nabla \mathcal{H}(\varphi)|^{2} d x d t=\kappa_{s}\|\varphi\|_{\mathscr{H}_{0}^{s}(E)}^{2} \geq \kappa_{s} \int_{E} b(x) \varphi^{2} d x \quad \forall \varphi \in C_{c}^{\infty}(E) .
$$

Proof. Put $g_{k}(x):=\min (b(x) u, k)>0$ for integers $k \geq 1$. Let $v_{k} \in \mathscr{H}_{0}^{s}(E)$ be the solution to

$$
\mathcal{B}_{s} v_{k}=g_{k} \quad \text { in } E \text {. }
$$

By Lemma 2.2, we have $\frac{1}{v_{k}} \in L_{l o c}^{\infty}(E)$ and by the standard maximum principle $\mathcal{H}\left(\widetilde{v_{k}}\right)>0$. Moreover by Lemma 2.5, we have

$$
u \geq v_{k}>0 \quad \text { in } E \text {. }
$$

Let $\varphi \in C_{c}^{\infty}(E)$. Put $V_{k}=\mathcal{H}\left(\widetilde{v_{k}}\right)$ and $V_{k}^{\varepsilon}=V_{k}+\varepsilon$, for $\varepsilon>0$. Set $\psi=\frac{\mathcal{H}(\varphi)}{V_{k}^{\varepsilon}}$ so that $V_{k}^{\varepsilon} \psi^{2} \in H_{0, T}^{1}\left(E ; t^{1-2 s}\right)$. Simple computations show that

$$
|\nabla \mathcal{H}(\varphi)|^{2}=\left|V_{k}^{\varepsilon} \nabla \psi\right|^{2}+\nabla V_{k}^{\varepsilon} \cdot \nabla\left(V_{k}^{\varepsilon} \psi^{2}\right)=\left|V_{k}^{\varepsilon} \nabla \psi\right|^{2}+\nabla V_{k} \cdot \nabla\left(V_{k}^{\varepsilon} \psi^{2}\right) .
$$

Thus using integration by parts we have

$$
\begin{aligned}
\int_{\mathbb{R}_{+}^{N+1}} t^{1-2 s}|\nabla \mathcal{H}(\varphi)|^{2} d x d t & \geq \int_{\mathbb{R}_{+}^{N+1}} t^{1-2 s} \nabla V_{k} \cdot \nabla\left(V_{k}^{\varepsilon} \psi^{2}\right) d x d t \\
& =\int_{E} g_{k} \frac{\varphi^{2}}{\left(v_{k}+\varepsilon\right)^{2}} d x .
\end{aligned}
$$

Take the limit as $\varepsilon \rightarrow 0$ to get

$$
\int_{\mathbb{R}_{+}^{N+1}} t^{1-2 s}|\nabla \mathcal{H}(\varphi)|^{2} d x d t \geq \kappa_{s} \int_{E} \frac{g_{k}}{v_{k}} \varphi^{2} d x
$$

by Fatou's lemma. By (2.21), we infer that

$$
\int_{\mathbb{R}_{+}^{N+1}} t^{1-2 s}|\nabla \mathcal{H}(\varphi)|^{2} d x d t \geq \kappa_{s} \int_{E} \frac{g_{k}}{u} \varphi^{2} d x
$$

Again by Fatou's lemma, inequality (2.20) follows immediately by taking $k \rightarrow+\infty$. 
The following result appeared in [3] in the case $s=1$.

Theorem 2.11 Let $E$ be a bounded Lipschitz domain of $\mathbb{R}^{N}$ with $0 \in E, N>2 s$. Then there is no nonnegative and nontrivial $u \in \mathcal{L}_{s}^{1}$ satisfying

$$
(-\Delta)^{s} u \geq \gamma|x|^{-2 s} u \quad \text { in } \mathcal{D}^{\prime}(E \backslash\{0\}),
$$

with $\gamma>\gamma_{0}$.

Proof. Note that $C_{s}(\{0\})=0$ provided $N>2 s$ (see [38, p. 397]). If such $u$ exits then $u>0$ in $E$ by the maximum principle thus Lemma 2.10 contradicts the sharpness of the Hardy constant $\gamma_{0}$.

\section{Nonexistence of positive supersolutions}

We start with the following

Lemma 3.1 For every $\alpha \in\left(-\frac{N}{2}-s, \frac{N}{2}-s\right)$, put $\vartheta_{\alpha}(x)=|x|^{\frac{2 s-N}{2}+\alpha}$. Then

$$
(-\Delta)^{s} \vartheta_{\alpha}=\gamma_{\alpha}|x|^{-2 s} \vartheta_{\alpha} \quad \text { in } \mathbb{R}^{N} \backslash\{0\}
$$

where

$$
\gamma_{\alpha}=2^{2 s} \frac{\Gamma\left(\frac{N+2 s+2 \alpha}{4}\right)}{\Gamma\left(\frac{N-2 s-2 \alpha}{4}\right)} \frac{\Gamma\left(\frac{N+2 s-2 \alpha}{4}\right)}{\Gamma\left(\frac{N-2 s+2 \alpha}{4}\right)} .
$$

For $\alpha \geq 0$, the function $\alpha \mapsto \gamma_{\alpha}$ is continuous and decreasing.

There exists a positive function $\Upsilon_{\alpha} \in C^{\beta}\left(\overline{\mathbb{R}_{+}^{N+1}} \backslash\{0\}\right)$ such that

$$
\left\{\begin{array}{l}
\operatorname{div}\left(t^{1-2 s} \nabla \Upsilon_{\alpha}\right)=0 \quad \text { in } \mathbb{R}_{+}^{N+1} \\
\Upsilon_{\alpha}=\vartheta_{\alpha} \quad \text { on } \partial \mathbb{R}_{+}^{N+1} \backslash\{0\} \\
-t^{1-2 s} \frac{\partial \Upsilon_{\alpha}}{\partial t}=\kappa_{s}(-\Delta)^{s} \vartheta_{\alpha}=\kappa_{s} \gamma_{\alpha}|x|^{-2 s} \vartheta_{\alpha} \quad \text { on } \partial \mathbb{R}_{+}^{N+1} \backslash\{0\}
\end{array}\right.
$$

Moreover if $\alpha>0$ then $\left|\nabla \Upsilon_{\alpha}\right| \in L^{2}\left(B_{+}^{N+1}(0, R) ; t^{1-2 s}\right)$ for every $R>0$.

Proof. Note that $\vartheta_{\alpha} \in \mathcal{L}_{s}^{1}$. The Fourier transform of radial functions (see [[44] Theorem 4.1]) yields

$$
\mathcal{F}\left(\vartheta_{\alpha}\right)(\rho)=\rho^{\frac{1-N}{2}} \int_{0}^{\infty}(r \rho)^{\frac{1}{2}} J_{\frac{N-2}{2}}(r \rho) \vartheta_{\alpha} r^{\frac{N-1}{2}} d r
$$


where $J_{\frac{N-2}{2}}$ is the Bessel function. Then we have

$$
\begin{aligned}
\mathcal{F}\left(\vartheta_{\alpha}\right)(\rho) & =\rho^{\frac{-N}{2}-s-\alpha} \int_{0}^{\infty}(r \rho)^{s+\alpha} J_{\frac{N-2}{2}}(r \rho) d(r \rho) \\
& =m_{\alpha} \rho^{\frac{-N}{2}-s-\alpha},
\end{aligned}
$$

where

$$
m_{\alpha}=2^{s+\alpha} \frac{\Gamma\left(\frac{N+2 s+2 \alpha}{4}\right)}{\Gamma\left(\frac{N-2 s-2 \alpha}{4}\right)} .
$$

Now we notice that $\left(\gamma_{\alpha}=m_{\alpha} m_{-\alpha}\right)$

$$
(-\Delta)^{s} \vartheta_{\alpha}=\mathcal{F}\left(\mathcal{F}\left((-\Delta)^{s} \vartheta_{\alpha}\right)\right)=\mathcal{F}\left(\rho^{2 s} \mathcal{F}\left(\vartheta_{\alpha}\right)(\rho)\right)=m_{\alpha} \mathcal{F}\left(\rho^{\frac{-N}{2}+s-\alpha}\right)=\gamma_{\alpha} r^{-2 s} \vartheta_{\alpha} .
$$

For the proof of the fact that the map $\alpha \mapsto \gamma_{\alpha}$ is continuous and decreasing, we refer to $[17]$.

We define

$$
\Upsilon_{\alpha}(t, x)=\left\{\begin{array}{l}
P(t, \cdot) * \vartheta_{\alpha}(t, x) \quad \forall(t, x) \in \mathbb{R}_{+}^{N+1} \\
\vartheta_{\alpha}(x) \quad \forall x \in \partial \mathbb{R}_{+}^{N+1} \backslash\{0\},
\end{array}\right.
$$

where $P$ is the Poisson kernel defined in Section 1.1. Clearly $\Upsilon_{\alpha}$ is positive. We have that

$$
-t^{1-2 s} \frac{\partial \Upsilon_{\alpha}}{\partial t}=\kappa_{s}(-\Delta)^{s} \vartheta_{\alpha} \quad \text { in } \mathbb{R}^{N} \backslash\{0\} .
$$

Hence we get (3.2).

From the regularity theory of $[11]$, we deduce that $\Upsilon_{\alpha} \in C^{\beta}\left(\overline{\mathbb{R}_{+}^{N+1}} \backslash\{0\}\right)$ for some $\beta>0$. In addition $\Upsilon_{\alpha} \in H^{1}\left(\Omega \times\left(t_{1}, t_{2}\right) ; t^{1-2 s}\right)$ for every $\Omega \subset \subset \mathbb{R}^{N} \backslash\{0\}$ and $0<t_{1}<t_{2}<\infty$.

Observe that $\Upsilon_{\alpha}(\lambda z)=\lambda^{\frac{2 s-N}{2}+\alpha} \Upsilon_{\alpha}(z)$ and thus choosing $\lambda=|z|^{-1}$, we infer that $\Upsilon_{\alpha}(z) \leq \Upsilon_{\alpha}\left(z|z|^{-1}\right)|z|^{\frac{2 s-N}{2}+\alpha} \leq C|z|^{\frac{2 s-N}{2}+\alpha}$, for every $z=(t, x) \in B_{+}^{N+1}(0, R)$ and $R>0$. From this, we deduce that $t^{1-2 s}|z|^{-2} \Upsilon_{\alpha}^{2} \in L^{1}\left(B_{+}^{N+1}(0, R)\right)$ for $\alpha>0$. We also have $|x|^{-2 s} \Upsilon_{\alpha}^{2} \in L_{l o c}^{1}\left(\mathbb{R}^{N}\right)$ for $\alpha>0$.

We let $\varphi$ be a cut-off function such that $\varphi=0$ for $|z|<\varepsilon, \varphi=1$ for $2 \varepsilon<|z|<R$, $\varphi=0$ for $|z|>2 R$ and $|\nabla \varphi| \leq C \varepsilon^{-1}$ for $\varepsilon<|z|<2 \varepsilon$. We use $\varphi^{2} \Upsilon_{\alpha}$ as a test function in (3.2) to get

$$
\int_{\mathbb{R}_{+}^{N+1}} t^{1-2 s} \nabla \Upsilon_{\alpha} \nabla\left(\varphi^{2} \Upsilon_{\alpha}\right)=\int_{\partial \mathbb{R}_{+}^{N+1}}|x|^{-2 s} \Upsilon_{\alpha}^{2} \varphi^{2}
$$


Integrating by parts and using Young's inequality, for some constant $c>0$, we have

$c \int_{B_{+}^{N+1}(0, R) \backslash B_{+}^{N+1}(0, \varepsilon)} t^{1-2 s} \varphi^{2}\left|\nabla \Upsilon_{\alpha}\right|^{2} \leq \int_{B^{N}(0,2 R)}|x|^{-2 s} \Upsilon_{\alpha}^{2} \varphi^{2}+\int_{\mathbb{R}_{+}^{N+1}} t^{1-2 s} \Upsilon_{\alpha}^{2}|\nabla \varphi|^{2}$.

Therefore

$c \int_{B_{+}^{N+1}(0, R) \backslash B_{+}^{N+1}(0, \varepsilon)} t^{1-2 s}\left|\nabla \Upsilon_{\alpha}\right|^{2} \leq \int_{B^{N}(0,2 R)}|x|^{-2 s} \Upsilon_{\alpha}^{2}+\int_{\varepsilon<|(t, x)|<2 \varepsilon} t^{1-2 s}|(t, x)|^{-2} \Upsilon_{\alpha}^{2}$.

Fatou's lemma yields $\left|\nabla \Upsilon_{\alpha}\right| \in L^{2}\left(B_{+}^{N+1}(0, R) ; t^{1-2 s}\right)$ for $\alpha>0$.

The comparison result obtained in Lemma 2.8 allows us to derive the following estimate when the potential $b(x)$ is the Hardy one.

Lemma 3.2 Let $N>2 s, \alpha \in[0,(N-2 s) / 2)$ and $p>1$. Suppose that $u \in$ $\mathcal{L}_{s}^{1} \cap L_{l o c}^{p}\left(B^{N}(0,2) \backslash\{0\}\right), u \nsupseteq 0$ such that

$$
(-\Delta)^{s} u-\gamma_{\alpha}|x|^{-2 s} u \geq u^{p} \quad \text { in } \mathcal{D}^{\prime}\left(B^{N}(0,2) \backslash\{0\}\right) .
$$

Then there exists a constant $C>0$ such that

$$
\|\varphi\|_{\mathscr{H}_{0}^{s}\left(B^{N}(0,2)\right)}^{2}-\gamma_{\alpha} \int_{B^{N}(0,2)}|x|^{-2 s} \varphi^{2} d x \geq C \int_{B^{N}(0,2)} \varphi^{2} d x \quad \forall \varphi \in C_{c}^{\infty}\left(B^{N}(0,2)\right) .
$$

Moreover there exists a constant $C^{\prime}>0$ such that

$$
u \geq v_{\alpha} \geq C^{\prime}|x|^{\frac{2 s-N}{2}+\alpha} \quad \text { in } B^{N}(0,1),
$$

where $v_{\alpha} \in \mathscr{H}_{0, b}^{s}\left(B^{N}(0,2)\right.$ ) (with $b(x)=\gamma_{\alpha}|x|^{-2 s}$ ) is the solution to

$$
\mathcal{B}_{s} v_{\alpha}-\gamma_{\alpha}|x|^{-2 s} v_{\alpha}=\min \left(u^{p}, 1\right) \quad \text { in } B^{N}(0,2) .
$$

Proof. Inequality (3.4) is trivial for $\alpha>0$ so we consider only the case $\alpha=0$. Since $C_{s}(\{0\})=0$ provided $N>2 s$, by Lemma 2.5 and Lemma 2.2, we have $u>0$ in $B^{N}(0,2)$ and

$$
M=e s s \inf _{B^{N}(0,1)} u>0 .
$$

Hence $u \in \mathcal{L}_{s}^{1}$ satisfies

$$
(-\Delta)^{s} u-\gamma_{\alpha}|x|^{-2 s} u \geq M^{p-1} u \quad \text { in } \mathcal{D}^{\prime}\left(B^{N}(0,1) \backslash\{0\}\right) .
$$


By Lemma 2.10 we obtain (3.4) thanks to the scale invariance of the integrals on the left hand side.

We put $b(x)=\gamma_{\alpha}|x|^{-2 s}$. By (3.4), for $\alpha \geq 0$, we can let $v_{\alpha} \in \mathscr{H}_{0, b}^{s}\left(B^{N}(0,2)\right)$ be the solution to

$$
\mathcal{B}_{s} v_{\alpha}-\gamma_{\alpha}|x|^{-2 s} v_{\alpha}=\min \left(u^{p}, 1\right) \quad \text { in } B^{N}(0,2) .
$$

Then by Lemma 2.8, we have $u \geq v_{\alpha}$ in $B^{N}(0,2)$. We first consider the case $\alpha>0$. Then $\gamma_{\alpha}<\gamma_{0}$ and thus $v_{\alpha} \in \mathscr{H}_{0}^{s}\left(B^{N}(0,2)\right)$. By the regularity result of [11], we get that $V_{\alpha}=\mathcal{H}\left(\widetilde{v_{\alpha}}\right)$ is continuous in $\overline{B_{+}^{N+1}(0,1)} \backslash\{0\}$ and $V_{\alpha} \geq 0$ by Lemma 2.3. Consider $\vartheta_{\alpha}$ and its harmonic extension $\Upsilon_{\alpha}$ given by Lemma 3.1. The maximum principal (see Lemma 2.2) implies that we can set

$$
C^{\prime}=\frac{\frac{\min }{S_{+}^{N}} v_{\alpha}}{\frac{\max }{\overline{S_{+}^{N}}} \Upsilon_{\alpha}}>0
$$

Put $w=C^{\prime} \Upsilon_{\alpha}-V_{\alpha}$. We have weakly

$$
\left\{\begin{array}{l}
\operatorname{div}\left(t^{1-2 s} \nabla w\right)=0 \quad \text { in } B_{+}^{N+1}(0,1), \\
w \leq 0 \quad \text { on } \overline{S_{+}^{N}}, \\
-t^{1-2 s} \frac{\partial w}{\partial t}-\kappa_{s} \gamma_{\alpha}|x|^{-2 s} w \leq 0 \quad \text { on } B^{N}(0,1) .
\end{array}\right.
$$

Then $w^{+}:=\max (w, 0) \in H_{0, S}^{1}\left(B_{+}^{N+1}(0,1) ; t^{1-2 s}\right)$ and therefore by integration by parts

$$
\int_{\mathbb{R}_{+}^{N+1}} t^{1-2 s}\left|\nabla w^{+}\right|^{2} d x d t-\kappa_{s} \gamma_{\alpha} \int_{B^{N}(0,1)}|x|^{-2 s}\left(w^{+}\right)^{2} d x \leq 0 .
$$

In particular

$$
\left\|w^{+}\right\|_{\mathscr{O}_{0}^{s}\left(B^{N}(0,1)\right)}^{2}-\gamma_{\alpha} \int_{B^{N}(0,1)}|x|^{-2 s}\left(w^{+}\right)^{2} d x \leq 0 .
$$

Hence $w^{+} \equiv 0$ by Hardy's inequality. Hence $v_{\alpha} \geq C^{\prime} \vartheta_{\alpha}$ in $B^{N}(0,1)$ that is $(3.5)$ for $\alpha>0$.

For the case $\alpha=0$, we put $\alpha_{n}=1 / n$ and we notice that the sequence $v_{\alpha_{n}} \in$ $\mathscr{H}_{0}^{s}\left(B^{N}(0,2)\right)$ solution to the problem

$$
\mathcal{B}_{s} v_{\alpha_{n}}-\gamma_{\alpha_{n}}|x|^{-2 s} v_{\alpha_{n}}=f \quad \text { in } B^{N}(0,2)
$$

is monotone increasing to $v_{0}$ because the mapping $\alpha \mapsto \gamma_{\alpha}$ is decreasing. Therefore, taking into account (3.6), we readily get (3.5). 


\section{Proof of Theorem 0.2}

Lemma 3.3 Let $E$ be a bounded Lipschitz domain of $\mathbb{R}^{N}, N>2 s$. Suppose that $0 \in E$ and $\alpha \in[0,(N-2 s) / 2)$. Let $u \in \mathcal{L}_{s}^{1} \cap L_{l o c}^{p}(E \backslash\{0\})$ such that

$$
(-\Delta)^{s} u-\gamma_{\alpha}|x|^{-2 s} u \geq u^{p} \quad \text { in } \mathcal{D}^{\prime}(E \backslash\{0\}),
$$

with

$$
\gamma_{\alpha}=2^{2 s} \frac{\Gamma\left(\frac{N+2 s+2 \alpha}{4}\right)}{\Gamma\left(\frac{N-2 s-2 \alpha}{4}\right)} \frac{\Gamma\left(\frac{N+2 s-2 \alpha}{4}\right)}{\Gamma\left(\frac{N-2 s+2 \alpha}{4}\right)} .
$$

If $p \geq \frac{N+2 s-2 \alpha}{N-2 s-2 \alpha}$, then $u=0$ in $E$.

Proof. Assume that $u \neq 0$. It follows from Lemma 3.2 that there exist $r, C_{r}>0$ such that

$$
u(x) \geq v_{\alpha}(x) \geq C_{r}|x|^{\frac{2 s-N}{2}+\alpha} \quad \forall x \in B^{N}(0, r) \subset E,
$$

where $v_{\alpha} \in \mathscr{H}_{0, b}^{s}(E)$ (with $b(x)=\gamma_{\alpha}|x|^{-2 s}$ ) is the solution to

$$
\mathcal{B}_{s} v_{\alpha}-\gamma_{\alpha}|x|^{-2 s} v_{\alpha}=\min \left(u^{p}, 1\right) \quad \text { in } E \text {. }
$$

On the other hand Lemma 2.10 yields, for all $\varphi \in C_{c}^{\infty}(E)$,

$$
\|\varphi\|_{\mathscr{H}_{0}^{s}(E)}^{2}-\gamma_{\alpha} \int_{E}|x|^{-2 s} \varphi^{2} d x \geq \int_{E} u^{p-1} \varphi^{2} d x .
$$

We first consider the case $\alpha>0$. If $r$ is small, by (3.9) we have, for $0<\alpha^{\prime}<\alpha$,

$$
(-\Delta)^{s} u-\gamma_{\alpha^{\prime}}|x|^{-2 s} u \geq\left(-\gamma_{\alpha^{\prime}}+\gamma_{\alpha}+C_{r}^{p-1}\right)|x|^{-2 s} u \quad \text { in } \mathcal{D}^{\prime}\left(B^{N}(0, r) \backslash\{0\}\right) .
$$

By Lemma 2.8 and using the same arguments as in Lemma 3.2 we get, provided $\alpha^{\prime} \nearrow \alpha$,

$$
u(x) \geq C_{r}^{\prime}|x|^{\frac{2 s-N}{2}+\alpha^{\prime}} \quad \forall x \in B^{N}(0, r / 2),
$$

for some constant $C_{r}^{\prime}>0$. Using the estimate (3.11) in (3.10) we get $\|\varphi\|_{\mathscr{O}_{0}^{s}\left(B^{N}(0, r / 2)\right)}^{2}-\gamma_{\alpha} \int_{B^{N}(0, r / 2)}|x|^{-2 s} \varphi^{2} d x \geq\left(C_{r}^{\prime}\right)^{p-1} \int_{B^{N}(0, r / 2)}|x|^{\left(\frac{2 s-N}{2}+\alpha^{\prime}\right)(p-1)} \varphi^{2} d x$, 
for all $\varphi \in C_{c}^{\infty}\left(B^{N}(0, r / 2)\right)$. Since $p>\frac{N+2 s-2 \alpha^{\prime}}{N-2 s-2 \alpha^{\prime}}$, we have

$$
-\delta:=\left(\frac{2 s-N}{2}+\alpha^{\prime}\right)(p-1)+2 s<0 .
$$

Hence for every $\rho \in(0, r / 2)$

$$
\|\varphi\|_{\mathscr{H}_{0}^{s}\left(B^{N}(0, \rho)\right)}^{2} \geq\left(\gamma_{\alpha}+\left(C_{r}^{\prime}\right)^{p-1} \rho^{-\delta}\right) \int_{B^{N}(0, \rho)}|x|^{-2 s} \varphi^{2} d x \quad \forall \varphi \in C_{c}^{\infty}\left(B^{N}(0, \rho)\right) .
$$

This contradicts the sharpness of the Hardy constant thanks to the scale invariance of the inequality.

Finally, for the case $\alpha=0$ we note that (3.10) implies, by density, that

$$
\left\|v_{\alpha}\right\|_{\mathscr{H}_{0}^{s}(E)}^{2}-\gamma_{\alpha} \int_{E}|x|^{-2 s} v_{\alpha}^{2} d x \geq \int_{E} v_{\alpha}^{p+1} d x .
$$

This also leads to a contradiction because $v_{\alpha} \in \mathscr{H}_{0, b}^{s}(E)$ while by (3.9)

$$
\begin{aligned}
\int_{E} v_{\alpha}^{p+1} d x & \geq C^{\prime} \int_{B^{N}(0, r)}|x|^{\left(\frac{2 s-N}{2}\right)(p+1)} d x \\
& \geq C^{\prime} \int_{S^{N-1}} \int_{0}^{r} t^{-1} d t d \sigma=+\infty
\end{aligned}
$$

for some constant $C^{\prime}>0$.

\section{Existence of positive solutions}

The proof will be separated into several cases. We put

$$
E_{\alpha}(u):=\|u\|_{\mathscr{H}_{0}^{s}(B)}^{2}-\gamma_{\alpha} \int_{B}|x|^{-2 s} u^{2} d x \quad \forall u \in C_{c}^{\infty}(B),
$$

where $B$ is a ball in $\mathbb{R}^{N}$ centered at 0 with $N>2 s$.

Case 1: $\alpha \in(0,(N-2 s) / 2]$ and $1<p<(N+s) /(N-2 s)$.

Thanks to the Hardy-Littlewood-Sobolev inequality and Hardy's inequality we have

$$
E_{\alpha}(u) \geq\left(\int_{B} u^{p+1}\right)^{2 /(p+1)} \quad \forall u \in C_{c}^{\infty}(B) .
$$


Thanks to the compact embedding of $\mathscr{H}_{0}^{s}(B)$ into $L^{p+1}(B)$, we can minimize $E_{\alpha}$ over the set

$$
\left\{u \in \mathscr{H}_{0}^{s}(B): \int_{B}\left(u^{+}\right)^{p+1}=1\right\}
$$

Let $u \in \mathscr{H}_{0}^{s}(B)$ be the minimizer. Put $u^{ \pm}=\max ( \pm u, 0)$. By Proposition 1.3, $u^{ \pm}$ belongs to $\mathscr{H}_{0}^{s}(B)$. We check rapidly that $E_{\alpha}\left(u^{+}\right) \leq E_{\alpha}(u)$. Observe that

$$
\kappa_{s}\left\|u^{ \pm}\right\|_{\mathscr{H}_{0}^{s}(B)}^{2}=\int_{\mathbb{R}_{+}^{N+1}} t^{1-2 s}\left|\nabla \mathcal{H}\left(u^{ \pm}\right)\right|^{2} d x d t \leq \int_{\mathbb{R}_{+}^{N+1}} t^{1-2 s}\left|\nabla\left(\mathcal{H}(u)^{ \pm}\right)\right|^{2} d x d t
$$

because $\mathcal{H}\left(u^{ \pm}\right)$and $\mathcal{H}(u)^{ \pm}$have the same trace on $\mathbb{R}^{N}$ while $\mathcal{H}\left(u^{ \pm}\right)$has minimal Dirichlet energy. Now using this and Hardy's inequality we have

$$
\begin{aligned}
\kappa_{s} E_{\alpha}\left(u^{+}\right)= & \kappa_{s}\left\|u^{+}\right\|_{\mathscr{H}_{0}^{s}(B)}^{2}-\kappa_{s} \gamma_{\alpha} \int_{B}|x|^{-2 s}\left(u^{+}\right)^{2} d x \\
= & \kappa_{s}\left\|u^{+}\right\|_{\mathscr{H}_{0}^{s}(B)}^{2}-\kappa_{s} \gamma_{\alpha} \int_{B}|x|^{-2 s} u^{2} d x+\kappa_{s} \gamma_{\alpha} \int_{B}|x|^{-2 s}\left(u^{-}\right)^{2} d x \\
\leq & \kappa_{s}\left\|u^{+}\right\|_{\mathscr{H}_{0}^{s}(B)}^{2}-\kappa_{s} \gamma_{\alpha} \int_{B}|x|^{-2 s} u^{2} d x+\kappa_{s}\left\|u^{-}\right\|_{\mathscr{H}_{0}^{s}(B)}^{2} \\
\leq & \int_{\mathbb{R}_{+}^{N+1}} t^{1-2 s}\left|\nabla\left(\mathcal{H}(u)^{+}\right)\right|^{2} d x d t-\kappa_{s} \gamma_{\alpha} \int_{B}|x|^{-2 s} u^{2} d x \\
& +\int_{\mathbb{R}_{+}^{N+1}} t^{1-2 s}\left|\nabla\left(\mathcal{H}(u)^{-}\right)\right|^{2} d x d t \\
= & \int_{\mathbb{R}_{+}^{N+1}} t^{1-2 s}|\nabla \mathcal{H}(u)|^{2} d x d t-\kappa_{s} \gamma_{\alpha} \int_{B}|x|^{-2 s} u^{2} d x \\
= & \kappa_{s} E_{\alpha}(u) .
\end{aligned}
$$

Thus we may assume that $u=u^{+}$is a nonegative and nontrivial minimizer therefore there exists a Lagrange multiplier $\lambda>0$ such that

$$
\mathcal{B}_{s} u-\gamma_{\alpha}|x|^{-2 s} u=\lambda u^{p} \quad \text { in } B .
$$

Hence $\lambda^{\frac{1}{p-1}} \widetilde{u}$ is a solution of problem (0.1).

Case 2: $\alpha=0$ and $1<p<(N+2 s) /(N-2 s)$.

Lemma 5.4 yields for every $q \in\left(2, \max \left(1, \frac{2}{1+2 s}\right)\right)$

$$
E_{0}(u) \geq\|u\|_{W_{0}^{\tau, q}(B)}^{2} \quad u \in C_{c}^{\infty}(B),
$$


with $\tau=\frac{1+2 s}{2}-\frac{1}{q}$. Therefore $\mathscr{H}_{0, b}^{s}(B)$ is compactly embedded into $L^{p+1}(B)$, with $b=\gamma_{0}|x|^{-2 s}$. Hence we can minimize $E_{0}$ over the set

$$
\left\{u \in \mathscr{H}_{0, b}^{s}(B): \int_{B}\left(u^{+}\right)^{p+1}=1\right\}
$$

We have to check again that $E_{0}\left(u^{+}\right) \leq E_{0}(u)$. But this can be done by density and using similar arguments as above. We skip the details. We get a positive minimizer

$u=u^{+}$of $E_{0}$ in the set (4.3). We conclude that $\lambda^{\frac{1}{p-1}} \widetilde{u}$ is a solution to (0.1) for some Lagrange multiplier $\lambda>0$.

Case 3: $\alpha \in(0,(N-2 s) / 2)$ and $(N+2 s) /(N-2 s) \leq p<(N+2 s-2 \alpha) /(N-2 s-2 \alpha)$. Consider $\vartheta_{\beta}=r^{\frac{2 s-N}{2}+\beta}$ given by Lemma 3.1 which satisfies

$$
(-\Delta)^{s} \vartheta_{\beta}=\gamma_{\beta}|x|^{-2 s} \vartheta_{\beta} \quad \text { in } \mathbb{R}^{N} \backslash\{0\}
$$

We look for a solution of the form $w=\mu r^{\frac{-2 s}{p-1}}$ with a constant $\mu>0$ to be determined in a minute. Assume that we can take $\beta \geq 0$ such that $r^{\frac{2 s-N}{2}+\beta}=r^{\frac{-2 s}{p-1}}$ then

$$
\begin{aligned}
(-\Delta)^{s} w & =\gamma_{\beta}|x|^{-2 s} w+w^{p}-w^{p-1} w \\
& =\gamma_{\alpha}|x|^{-2 s} w+w^{p}+\left(\gamma_{\beta}-\gamma_{\alpha}-\mu^{p-1}\right)|x|^{-2 s} w .
\end{aligned}
$$

Since $\beta \mapsto \gamma_{\beta}$ is decreasing, we can choose $\mu^{p-1}=\gamma_{\beta}-\gamma_{\alpha}>0$ provided $\alpha>\beta$. But note that $\alpha>\beta$ as soon as $p<(N+2 s-2 \alpha) /(N-2 s-2 \alpha)$ and $p \geq(N+2 s) /(N-2 s)$ implies $\beta \geq 0$. In conclusion we have, in $\mathbb{R}^{N} \backslash\{0\}$,

$$
(-\Delta)^{s} w-\gamma_{\alpha}|x|^{-2 s} w=w^{p}
$$

and $w \in \mathcal{L}_{s}^{1} \cap L^{p}(B)$ is a solution to $(0.1)$.

\section{Appendix}

\subsection{Remainder term for the fractional Hardy inequality}

Let $E$ be a bounded open set of $\mathbb{R}^{N}, N>2 s$, with $0 \in E$. The following (local) Hardy inequality is a consequence of $(0.4)$

$$
\gamma_{0} \int_{E} u^{2}|x|^{-2 s} d x \leq\|u\|_{\mathscr{H}_{0}^{s}(E)}^{2} \quad \forall u \in C_{c}^{\infty}(E)
$$


In addition the constant $\gamma_{0}$ is optimal. Our objective, in this section, is to improve inequality (0.4) in bounded domains of $\mathbb{R}^{N}$.

Many deal of work has been done in improving the classical Hardy inequality starting from the work of Brezis-Vázquez [10]. We also quote [4], [46], [27] for related improvements.

We shall prove a Vázquez-Zuazua-type (see [46]) improvement for the fractional Hardy inequality (0.4). That is for $2>q>\max \left(1, \frac{2}{2-a}\right)$, there exists a constant $C(E)>0$ such that for all $u \in C_{c}^{\infty}(E)$,

$$
C(E)\|u\|_{W_{0}^{\tau, q}(E)}^{2} \leq\|u\|_{\mathscr{H}_{0}^{s}(E)}^{2}-\gamma_{0} \int_{E}|x|^{a-1} u^{2} d x,
$$

where $a=1-2 s$ and $\tau=\frac{2-a}{2}-\frac{1}{q}$. The proof requires several preliminary lemmata.

Consider the function $\Upsilon_{0}$ defined in Lemma 3.1 satisfying

$$
\left\{\begin{array}{l}
\operatorname{div}\left(t^{1-2 s} \Upsilon_{0}\right)=0 \quad \text { in } \mathbb{R}_{+}^{N+1}, \\
\Upsilon_{0}=|x|^{\frac{2 s-N}{2}} \quad \text { on } \mathbb{R}^{N} \backslash\{0\}, \\
-t^{1-2 s} \frac{\partial \Upsilon_{0}}{\partial t}=\kappa_{s} \gamma_{0}|x|^{-2 s} \Upsilon_{0} \quad \text { on } \mathbb{R}^{N} \backslash\{0\}
\end{array}\right.
$$

We have seen in the proof of Lemma 3.1 that

$$
\left|\Upsilon_{0}(z)\right| \leq C|z|^{(2 s-N) / 2} \quad \forall z=(x, t) \in B_{+}^{N+1} .
$$

By scale invariance, we have that

$$
\Upsilon_{0}(z)=R^{(N-2 s) / 2} \Upsilon_{0}(R z) \quad \forall R>0
$$

This implies the estimate

$$
\left|\Upsilon_{0}(z)\right| \geq C|z|^{(2 s-N) / 2} \quad \forall z \in B_{+}^{N+1}
$$

and also

$$
\left|\nabla \Upsilon_{0}(z)\right| \leq C|z|^{(2 s-N) / 2-1} \quad \forall z \in B_{+}^{N+1}
$$

We now prove the following result which were proved in [17] when $s=1 / 2$. 
Lemma 5.1 For every $q \in(1,2)$ there exists a constant $C>0$ such that for all $\varphi \in C_{c}^{\infty}\left(B^{N+1}\right)$

$$
C\left(\int_{B_{+}^{N+1}} t^{\frac{q a}{2}}|\nabla \varphi|^{q} d z\right)^{2 / q} \leq \int_{B_{+}^{N+1}} t^{a}|\nabla \varphi|^{2} d z-\kappa_{s} \gamma_{0} \int_{B^{N}}|x|^{a-1} \varphi^{2} d x
$$

where $a=1-2 s$.

Proof. Let $\varphi \in C_{c}^{\infty}\left(B^{N+1} \backslash\{0\}\right)$ and put $\psi=\frac{\varphi}{\Upsilon_{0}}$. Simple computations yield

$$
|\nabla \varphi|^{2}=\left|\Upsilon_{0} \nabla \psi\right|^{2}+\nabla \Upsilon_{0} \cdot \nabla\left(\Upsilon_{0} \psi^{2}\right)
$$

Integration by parts and using (5.2) leads to

$$
\int_{B_{+}^{N+1}} t^{a}|\nabla \varphi|^{2} d z-\kappa_{s} \gamma_{0} \int_{B^{N}}|x|^{a-1} \varphi^{2} d x \geq \int_{B_{+}^{N+1}} t^{a} \Upsilon_{0}^{2}|\nabla \psi|^{2} d z
$$

By (5.5) and using polar coordinates $z=r \sigma=|z| \frac{z}{|z|}$, we get

$$
\int_{B_{+}^{N+1}} t^{a} \Upsilon_{0}^{2}|\nabla \psi|^{2} d z \geq C \int_{0}^{1} \int_{S_{+}^{N}}\left(\sigma_{1}\right)^{a} r|\nabla \psi|^{2} d \sigma d r
$$

where $\sigma_{1}$ is the component of $\sigma$ in the $t$ direction. We wish to show that there exists a constant $C>0$ such that

$$
I:=\left(\int_{0}^{1} \int_{S_{+}^{N}}\left(\sigma_{1}\right)^{a} r|\nabla \psi|^{2} d \sigma d r\right)^{q / 2} \geq C \int_{B_{+}^{N+1}} t^{\frac{q a}{2}}|\nabla \varphi|^{q} d z \quad \forall \varphi \in C_{c}^{\infty}\left(B^{N+1}\right) .
$$

We have

$\int_{B_{+}^{N+1}} t^{\frac{q a}{2}}|\nabla \varphi|^{q} d z=\int_{B_{+}^{N+1}} t^{\frac{q a}{2}}\left|\nabla \psi \Upsilon_{0}+\psi \nabla \Upsilon_{0}\right|^{q} d z \leq C \int_{B_{+}^{N+1}} t^{\frac{q a}{2}}\left(\left|\nabla \psi \Upsilon_{0}\right|^{q}+\left|\psi \nabla \Upsilon_{0}\right|^{q}\right) d z$

Put

$$
\begin{aligned}
& I_{1}=\int_{B_{+}^{N+1}} t^{\frac{q a}{2}}\left|\nabla \psi \Upsilon_{0}\right|^{q} d z \\
& I_{2}=\int_{B_{+}^{N+1}} t^{\frac{q a}{2}}\left|\psi \nabla \Upsilon_{0}\right|^{q} d z
\end{aligned}
$$


Using (5.3) and Hölder inequality, we have

$$
\begin{aligned}
I_{1} & \leq C \int_{S_{+}^{N}}\left(\sigma_{1}\right)^{q a / 2} \int_{0}^{1} r^{N+q(1-N) / 2}|\nabla \psi|^{q} d r d \sigma \\
& =C \int_{0}^{1} r^{N+q(1-N) / 2-q / 2} \int_{S_{+}^{N}}\left(\sigma_{1}\right)^{q a / 2} r^{q / 2}|\nabla \psi|^{q} d \sigma d r \\
& \leq C \int_{0}^{1} r^{N(2-q) / 2}\left(\int_{S_{+}^{N}}\left(\sigma_{1}\right)^{a} r|\nabla \psi|^{2} d \sigma\right)^{q / 2} d r \\
& \leq C \int_{0}^{1}\left(\int_{S_{+}^{N}}\left(\sigma_{1}\right)^{a} r|\nabla \psi|^{2} d \sigma\right)^{q / 2} d r \\
& \leq C\left(\int_{0}^{1} \int_{S_{+}^{N}}\left(\sigma_{1}\right)^{a} r|\nabla \psi|^{2} d \sigma d r\right)^{q / 2} \\
& \leq C I .
\end{aligned}
$$

On the other hand we have using (5.6)

$$
\begin{aligned}
I_{2} \leq & C \int_{S_{+}^{N}}\left(\sigma_{1}\right)^{q a / 2} \int_{0}^{1} r^{N(2-q) / 2-q / 2}|\psi|^{q} d r d \sigma \\
& \leq C \int_{S_{+}^{N}}\left(\sigma_{1}\right)^{q a / 2} \int_{0}^{1} r^{N(2-q) / 2-q / 2+q}\left|\frac{\partial \psi}{\partial r}\right|^{q} d r d \sigma \\
& \leq C \int_{0}^{1} r^{N+q(1-N) / 2-q / 2} \int_{S_{+}^{N}}\left(\sigma_{1}\right)^{q a / 2} r^{q / 2}|\nabla \psi|^{q} d \sigma d r \\
& \leq C I
\end{aligned}
$$

where in the second inequality we have used the one dimensional Hardy inequality $\int_{0}^{1} f^{q} d r \leq c \int_{0}^{1} r^{-q}\left|f^{\prime}\right|^{q} d r$ and observing that (5.10) is just (5.11). The lemma follows because $C_{c}^{\infty}\left(B^{N+1} \backslash\{0\}\right)$ is dense in $C_{c}^{\infty}\left(B^{N+1}\right)$ with respect to the $H^{1}\left(B^{N+1} ; t^{a}\right)$ norm when $N \geq 3$, see [30].

The Lion's interpolation inequality, [[34] Paragraph 5], shows that for $a \neq 0$ and $-\frac{1}{q}<\frac{a}{2}<\frac{1}{q}$ there exits a constant $C>0$ such that

$$
C\|v\|_{W^{\tau, q}\left(\mathbb{R}^{N}\right)}^{q} \leq \int_{\mathbb{R}_{+}^{N+1}} t^{\frac{a q}{2}}|\nabla v|^{q} d x d t+\int_{\mathbb{R}_{+}^{N+1}} t^{\frac{a q}{2}}|v|^{q} d x d t, \quad \forall v \in C_{c}^{\infty}\left(\mathbb{R}^{N+1}\right),
$$


where $\tau=\frac{2-a}{2}-\frac{1}{q}$.

We first prove a generalized weighted Poincaré trace inequality. The proof is standard. We recall that the space $W_{0, S}^{1, q}\left(B_{+}^{N+1} ; t^{q a / 2}\right)$ was defined in Section 1.

Lemma 5.2 Suppose that $q>1$ and $\tau:=\frac{2-a}{2}-\frac{1}{q}>0$. Then the following inequality holds

$\int_{B_{+}^{N+1}} t^{q a / 2}|u|^{q} d z \leq C \int_{B_{+}^{N+1}} t^{q a / 2}|\nabla u|^{q} d z+C \int_{B^{N}}|u|^{q} d z \quad \forall u \in W_{0, S}^{1, q}\left(B_{+}^{N+1} ; t^{q a / 2}\right)$.

Proof. Inequality (5.13) is well known for $a=0$. So we restrict ourself to the case $a \neq 0$.

Assume by contradiction that (5.13) does not hold. Then there exits a sequence $u_{n} \in W_{0, S}^{1, q}\left(B_{+}^{N+1} ; t^{q a / 2}\right)$ such that

$$
\int_{B_{+}^{N+1}} t^{q a / 2}\left|\nabla u_{n}\right|^{q} d z+\int_{B^{N}}\left|u_{n}\right|^{q} d z=o(1)
$$

and

$$
\int_{B_{+}^{N+1}} t^{q a / 2}\left|u_{n}\right|^{q} d z>0 \quad \forall n \in \mathbb{N} .
$$

Up to normalization, we may assume that $\int_{B_{+}^{N+1}} t^{q a / 2}\left|u_{n}\right|^{q} d z=1$. But then (5.14) implies that $u_{n}$ is bounded in $W^{1, q}\left(B_{+}^{N+1} ; t^{q a / 2}\right)$ thus $u_{n} \rightarrow u$ in $W^{1, q}\left(B_{+}^{N+1} ; t^{q a / 2}\right)$ and $u_{n} \rightarrow u$ in $L^{q}\left(B_{+}^{N+1} ; t^{q a / 2}\right)$ (see [26]) so that

$$
\int_{B_{+}^{N+1}} t^{q a / 2}|u|^{q} d z=1 .
$$

It follows from (5.12) and the compact embedding of $W_{0}^{\tau, q}\left(B^{N}\right)$ into $L^{q}\left(B^{N}\right)$, that $u_{n} \rightarrow u$ in $L^{q}\left(B^{N}\right)$. From $(5.14)$ we get $\left.u\right|_{\mathbb{R}^{N}}=0$ and also $\nabla u=0$. It turns out that $u=0$ in $B_{+}^{N+1}$, a contradiction with (5.15).

By an argument of partition of unity, we have the following

Lemma 5.3 Let $2>q>\max \left(1, \frac{2}{2-a}\right)$. There exist some constants $C, c>0$ such that for all $\varphi \in C_{c}^{\infty}\left(\mathbb{R}^{N+1}\right)$

$$
C\|\varphi\|_{W^{\tau, q}\left(B^{N}\right)}^{2} \leq \int_{\mathbb{R}_{+}^{N+1}} t^{a}|\nabla \varphi|^{2} d z-\kappa_{s} \gamma_{0} \int_{\mathbb{R}^{N}}|x|^{a-1} \varphi^{2} d x+c \int_{\mathbb{R}^{N}} \varphi^{2} d x,
$$

where $a=1-2 s$ and $\tau=\frac{2-a}{2}-\frac{1}{q}$. 
Proof. We put

$$
J(v)=\int_{\mathbb{R}_{+}^{N+1}} t^{a}|\nabla v|^{2} d z-\kappa_{s} \gamma_{0} \int_{\mathbb{R}^{N}}|x|^{a-1} v^{2} d x
$$

Let $\chi \in C_{c}^{\infty}\left(B^{N+1}\right), 0 \leq \chi \leq 1$ in $B^{N+1}$ and such that $\chi \equiv 1$ on $B^{N+1}(0,1 / 2)$. Let $\eta \in H^{1}\left(B_{+}^{N+1} ; t^{a}\right)$ be the minimum of the problem

$$
\inf \left\{\int_{B_{+}^{N+1}} t^{a}|\nabla u|^{2} d z: u-\chi \in H_{0}^{1}\left(B_{+}^{N+1} ; t^{a}\right)\right\} .
$$

Then

$$
\begin{cases}\operatorname{div}\left(t^{a} \nabla \eta\right)=0 \quad \text { in } B_{+}^{N+1} \\ \eta=\chi & \text { on } B^{N} \\ \eta=0 & \text { on } \overline{S_{+}^{N}}\end{cases}
$$

It turns out that $0 \leq \eta \leq 1$ in $B_{+}^{N+1}$. In addition, thanks to [14], $\lim _{t \rightarrow 0} t^{a} \frac{\partial \eta}{\partial t} \in$ $L_{\text {loc }}^{\infty}\left(B^{N}\right)$. Given $\varphi \in C_{c}^{\infty}\left(\mathbb{R}^{N+1}\right)$, simple computations based on integration by parts lead to

$$
\int_{\mathbb{R}_{+}^{N+1}} t^{a}|\nabla(\varphi \eta)|^{2} d z \leq \int_{\mathbb{R}_{+}^{N+1}} t^{a}|\nabla \varphi|^{2} d z+c \int_{B^{N}} \varphi^{2} d x
$$

where $c>0$ depends only on $\eta$. On the other hand we have

$$
\begin{aligned}
\int_{B^{N}}|x|^{a-1}(\eta \varphi)^{2} d x & =\int_{\mathbb{R}^{N}}|x|^{a-1} \varphi^{2} d x+\int_{\mathbb{R}^{N}}\left(1-\eta^{2}\right)|x|^{a-1} \varphi^{2} \\
& \leq \int_{\mathbb{R}^{N}}|x|^{a-1} \varphi^{2} d x+c \int_{\mathbb{R}^{N}} \varphi^{2} d x
\end{aligned}
$$

Therefore we obtain

$$
J(\varphi \eta) \leq J(\varphi)+c \int_{\mathbb{R}^{N}} \varphi^{2} d x
$$

Applying Lemma 5.1, we infer that

$$
\left(\int_{\mathbb{R}_{+}^{N+1}} t^{\frac{q a}{2}}|\nabla(\eta \varphi)|^{q} d z\right)^{2 / q} \leq J(\varphi)+c \int_{\mathbb{R}^{N}} \varphi^{2} d x .
$$

By Lemma 5.2 and Hölder inequality $(1<q<2)$

$$
C\|\eta \varphi\|_{W^{1, q}\left(\mathbb{R}_{+}^{N+1} ; t^{q a / 2}\right)}^{2} \leq J(\varphi)+c \int_{\mathbb{R}^{N}} \varphi^{2} d x .
$$


Using Lions' interpolation inequality (5.12) with $a \neq 0$, we obtain

$$
C\|\eta \varphi\|_{W^{\tau, q}\left(\mathbb{R}^{N}\right)}^{2} \leq J(\varphi)+c \int_{\mathbb{R}^{N}} \varphi^{2} d x
$$

If $a=0$, it is well know that $W^{1, q}\left(\mathbb{R}_{+}^{N+1}\right)$ embeds continuously into $W^{1-1 / q, q}\left(\mathbb{R}^{N}\right)$. Recalling that $\eta=\chi \equiv 1$ on $B^{N}(0,1 / 2)$, the lemma follows by scaling.

Taking advantages to the singular nature of the Hardy potential and the scale invariance, we prove the main result in this section:

Lemma 5.4 Let $2>q>\max \left(1, \frac{2}{2-a}\right)$. Then there exists a constant $C_{0}>0$ such that for all $u \in C_{c}^{\infty}\left(B^{N}\right)$,

$$
C_{0}\|u\|_{W_{0}^{\tau, q}\left(B^{N}\right)}^{2} \leq\|u\|_{\mathscr{H}_{0}^{s}\left(B^{N}\right)}^{2}-\gamma_{0} \int_{B^{N}}|x|^{a-1} u^{2} d x
$$

where $a=1-2 s$ and $\tau=\frac{2-a}{2}-\frac{1}{q}$.

Proof. Let $u \in C_{c}^{\infty}\left(B^{N}\right)$ and we define $U=\mathcal{H}(\widetilde{u})=\mathcal{H}(u)$. Then $U \in H^{1}\left(\mathbb{R}_{+}^{N+1} ; t^{a}\right)$ thus by Lemma 5.3 and a density argument we get

$$
C\|U\|_{W^{\tau, q}\left(B^{N}\right)}^{2} \leq \int_{\mathbb{R}_{+}^{N+1}} t^{a}|\nabla U|^{2} d z-\kappa_{s} \gamma_{0} \int_{\mathbb{R}^{N}}|x|^{a-1} U^{2} d x+c \int_{\mathbb{R}^{N}} U^{2} d x .
$$

Since $u=U$ on $B^{N}$, it follows that

$$
C\|u\|_{W^{\tau, q}\left(B^{N}\right)}^{2} \leq\|u\|_{\mathscr{H}_{0}^{s}\left(B^{N}\right)}^{2}-\gamma_{0} \int_{B^{N}}|x|^{a-1} u^{2} d x+c \int_{B^{N}} u^{2} d x .
$$

Let $r \in(0,1)$ we derive from the above that for every $u \in C_{c}^{\infty}\left(B^{N}(0, r)\right)$

$$
C\|u\|_{W^{\tau, q}\left(B^{N}(0, r)\right)}^{2} \leq\|u\|_{\mathscr{H}_{0}^{s}\left(B^{N}(0, r)\right)}^{2}-\gamma_{0} \int_{B^{N}(0, r)}|x|^{a-1} u^{2} d x+c \int_{B^{N}(0, r)} u^{2} d x,
$$

with $c, C>0$ independent on $r$. This holds because $\|u\|_{\mathscr{H}_{0}^{s}\left(B^{N}(0, r)\right)}=\|u\|_{\mathscr{H}_{0}^{s}\left(B^{N}(0,1)\right)}$ as long as $u \in C_{c}^{\infty}\left(B^{N}(0, r)\right)$ and $r<1$.

It is clear from (5.18) that wee need only to show that there exists a constant $C_{2}>0$ such that for every $u \in C_{c}^{\infty}\left(B^{N}\right)$

$$
C_{2} \int_{B^{N}} u^{2} d x \leq\|u\|_{\mathscr{H}_{0}^{s}\left(B^{N}\right)}^{2}-\gamma_{0} \int_{B^{N}}|x|^{a-1} u^{2} d x .
$$


Before proceeding, we recall that the mapping $\alpha \mapsto \gamma_{\alpha}$ (defined in Lemma 3.1) is decreasing. We will use this fact and the estimates in Lemma 3.2 to conclude the proof. Pick

$$
\alpha \in\left(0, \frac{N-2 s}{2}\right)
$$

and let $r>0$ be so small that

$$
\left(\gamma_{0}-\gamma_{\alpha}\right) r^{a-1}-c>0 .
$$

As we did in Section 4, by (5.18), we can define the space $\mathscr{H}_{0, b}^{s}\left(B^{N}(0, r)\right)$ with $b(x)=\gamma_{0}|x|^{a-1}-c$. Letting $2<p+1<\frac{2 N}{N-2 s}$, we can choose $q$ (close to 2 ) so that $W_{0}^{\tau, q}\left(B^{N}(0, r)\right)$ is compactly embedded into $L^{p+1}\left(B^{N}(0, r)\right)$. Then minimization procedure implies that there exits a nonnegative and nontrivial $u_{r} \in \mathscr{H}_{0, b}^{s}\left(B^{N}(0, r)\right)$ solution to

$$
\mathcal{B}_{s} u_{r}+c u_{r}-\gamma_{0}|x|^{a-1} u_{r}=C u_{r}^{p} \quad \text { in } B^{N}(0, r) .
$$

By density

$$
(-\Delta)^{s} \widetilde{u_{r}}+c \widetilde{u_{r}}-\gamma_{0}|x|^{a-1} \widetilde{u_{r}}=C{\widetilde{u_{r}}}^{p} \quad \text { in } \mathcal{D}^{\prime}\left(B^{N}(0, r)\right) .
$$

We have, by (5.20),

$$
(-\Delta)^{s} \widetilde{u_{r}}-\gamma_{\alpha}|x|^{a-1} \widetilde{u_{r}} \geq\left(\left(\gamma_{0}-\gamma_{\alpha}\right) r^{a-1}-c\right) \widetilde{u_{r}}+C{\widetilde{u_{r}}}^{p} \geq C{\widetilde{u_{r}}}^{p} \quad \text { in } \mathcal{D}^{\prime}\left(B^{N}(0, r)\right) \text {. }
$$

Therefore by Lemma 3.2, there exists a constant $C_{r}>0$ such that

$$
u_{r} \geq C_{r}|x|^{-\frac{N-2 s}{2}+\alpha} \quad \text { in } B^{N}(0, r / 2) \text {. }
$$

For every $k \in \mathbb{N}$, take $v^{k} \in \mathscr{H}_{0}^{s}\left(B^{N}(0, r)\right)$ as the solution to

$$
\mathcal{B}_{s} v_{k}+c v_{k}-\left(\gamma_{0}-1 / k\right)|x|^{a-1} v_{k}=C \min \left(u_{r}^{p}, k\right) \quad \text { in } B^{N}(0, r) .
$$

Since $\widetilde{u_{r}}$ satisfies (5.21), it follows (see Remark 2.9) that

$$
u_{r} \geq v_{k}>0 \quad \text { in } B^{N}(0, r)
$$

by Lemma 2.3 and Lemma 2.2 .

Put $V_{k}=\mathcal{H}\left(\widetilde{v_{k}}\right)$ we have that for any $\Psi \in H_{0, T}^{1}\left(B^{N}(0, r) ; t^{a}\right)$

$$
\begin{aligned}
\kappa_{s}^{-1} \int_{\mathbb{R}_{+}^{N+1}} t^{a} \nabla V_{k} \cdot \nabla \Psi d z=\left(\gamma_{0}-1 / k\right) & \int_{B^{N}(0, r)}|x|^{a-1} v_{k} \Psi d x-c \int_{B^{N}(0, r)} v_{k} \Psi d x \\
& +C \int_{B^{N}(0, r)} \min \left(u_{r}^{p}, k\right) \Psi d x .
\end{aligned}
$$


Thanks to (5.22), we can choose $r^{\prime} \in(0, r / 2)$ (small) such that

$$
-c+C u_{r}^{p-1} \geq 1 \quad \text { in } B^{N}\left(0, r^{\prime}\right)
$$

For such a fixed $r^{\prime}$, take $\varphi \in C_{c}^{\infty}\left(B^{N}\left(0, r^{\prime}\right)\right)$. For $\varepsilon>0$, set $V_{k}^{\varepsilon}=V_{k}+\varepsilon$ and put $\psi:=\frac{\mathcal{H}(\varphi)}{V_{k}^{\varepsilon}}$. We have

$$
|\nabla \mathcal{H}(\varphi)|^{2}=\left|V_{k}^{\varepsilon} \nabla \psi\right|^{2}+\nabla V_{k} \cdot \nabla\left(V_{k}^{\varepsilon} \psi^{2}\right)
$$

and also $V_{k}^{\varepsilon} \psi^{2} \in H_{0, T}^{1}\left(B^{N}(0, r) ; t^{a}\right)$. This together with (5.24) yields

$$
\begin{aligned}
\kappa_{s}^{-1} \int_{\mathbb{R}_{+}^{N+1}} t^{a}|\nabla \mathcal{H}(\varphi)|^{2} d x d t \geq & \left(\gamma_{0}-1 / k\right) \int_{B^{N}\left(0, r^{\prime}\right)}|x|^{a-1} \frac{v_{k}}{v_{k}+\varepsilon} \varphi^{2} d x \\
& -c \int_{B^{N}\left(0, r^{\prime}\right)} \frac{v_{k}}{v_{k}+\varepsilon} \varphi^{2} d x \\
& +C \int_{B^{N}\left(0, r^{\prime}\right)} \frac{\min \left(u_{r}^{p}, k\right)}{v_{k}+\varepsilon} \varphi^{2} d x .
\end{aligned}
$$

Thus

$$
\begin{aligned}
\kappa_{s}^{-1} \int_{\mathbb{R}_{+}^{N+1}} t^{a}|\nabla \mathcal{H}(\varphi)|^{2} d x d t \geq & \left(\gamma_{0}-1 / k\right) \int_{B^{N}\left(0, r^{\prime}\right)}|x|^{a-1} \frac{v_{k}}{v_{k}+\varepsilon} \varphi^{2} d x-c \int_{B^{N}\left(0, r^{\prime}\right)} \varphi^{2} d x \\
& +C \int_{B^{N}\left(0, r^{\prime}\right)} \frac{\min \left(u_{r}^{p}, k\right)}{v_{k}+\varepsilon} \varphi^{2} d x .
\end{aligned}
$$

By Fatou's lemma, when $\varepsilon \rightarrow 0$, we have

$$
\begin{aligned}
\kappa_{s}^{-1} \int_{\mathbb{R}_{+}^{N+1}} t^{a}|\nabla \mathcal{H}(\varphi)|^{2} d x d t \geq & \left(\gamma_{0}-1 / k\right) \int_{B^{N}\left(0, r^{\prime}\right)}|x|^{a-1} \varphi^{2} d x-c \int_{B^{N}\left(0, r^{\prime}\right)} \varphi^{2} d x \\
& +C \int_{B^{N}\left(0, r^{\prime}\right)} \frac{\min \left(u_{r}^{p}, k\right)}{v_{k}} \varphi^{2} d x .
\end{aligned}
$$

By (5.23) we obtain

$$
\begin{aligned}
\kappa_{s}^{-1} \int_{\mathbb{R}_{+}^{N+1}} t^{a}|\nabla \mathcal{H}(\varphi)|^{2} d x d t \geq & \left(\gamma_{0}-1 / k\right) \int_{B^{N}\left(0, r^{\prime}\right)}|x|^{a-1} \varphi^{2} d x-c \int_{B^{N}\left(0, r^{\prime}\right)} \varphi^{2} d x \\
& +C \int_{B^{N}\left(0, r^{\prime}\right)} \frac{\min \left(u_{r}^{p}, k\right)}{u_{r}} \varphi^{2} d x .
\end{aligned}
$$


It follows again from Fatou's lemma that

$$
\kappa_{s}^{-1} \int_{\mathbb{R}_{+}^{N+1}} t^{a}|\nabla \mathcal{H}(\varphi)|^{2} d x d t \geq \gamma_{0} \int_{B^{N}\left(0, r^{\prime}\right)}|x|^{a-1} \varphi^{2} d x+\int_{B^{N}\left(0, r^{\prime}\right)}\left(-c+C u_{r}^{p-1}\right) \varphi^{2} d x .
$$

From the choice of $r^{\prime}$ in (5.25), we get immediately, for any $\varphi \in C_{c}^{\infty}\left(B^{N}\left(0, r^{\prime}\right)\right)$,

$$
\kappa_{s}^{-1} \int_{\mathbb{R}_{+}^{N+1}} t^{a}|\nabla \mathcal{H}(\varphi)|^{2} d x d t-\gamma_{0} \int_{B^{N}\left(0, r^{\prime}\right)}|x|^{a-1} \varphi^{2} d x \geq \int_{B^{N}\left(0, r^{\prime}\right)} \varphi^{2} d x .
$$

By scaling we have (5.19) which was our objective.

\section{Acknowledgments}

This work is supported by the Alexander-von-Humboldt Foundation. The author would like to thank Professor Tobias Weth for fruitful discussions.

\section{References}

[1] S. Armstrong, B. Sirakov, Nonexistence of positive supersolutions of elliptic equations via the maximum principle. Comm. PDE, to appear.

[2] S. Armstrong, B. Sirakov, A new approach to Liouville theorems for elliptic inequalities. RIMS proceeding, to appear.

[3] P. Baras and J. A. Goldstein, The heat equation with a singular potential, Trans. Amer. Math. Soc. 284 (1984), no. 1, 121-139.

[4] G. Barbatis, S. Filippas, A. Tertikas, A unified approach to improved $L^{p}$ Hardy inequalities with best constants . Trans. Amer. Math. Soc., 356, (2004), 2169-2196.

[5] K. Bogdan; K. Burdzy; Z-Q. Chen, Censored stable processes. Probab. Theory Related Fields 127 (2003), no. 1, 89-152.

[6] H. Brezis and X. Cabré. Some simple nonlinear PDEs without solutions. Bull. UMI 1 (1998), 223-262.

[7] H. Brezis, L. Dupaigne, A. Tesei, On a semilinear elliptic equation with inversesquare potential. Selecta Math. (N.S.) 11 (2005), no. 1, 1-7. 
[8] H. Brezis and M. Marcus, Hardy's inequalities revisited. Dedicated to Ennio De Giorgi. Ann. Scuola Norm. Sup. Pisa Cl. Sci. (4) 25 (1997), no. 1-2, 217-237.

[9] H. Brezis, M. Marcus and I. Shafrir, Extermal functions for Hardy's inequality with weight, J. Funct. Anal. 171 (2000), 177-191.

[10] H. Brezis and J. L. Vázquez, Blow-up solutions of some nonlinear elliptic problems, Rev. Mat. Univ. Complut. Madrid 10 (1997), no. 2, 443-469.

[11] X. Cabré and Y. Sire, Nonlinear equations for fractional Laplacians I: Regularity, maximum principles, and Hamiltonian estimates (2010).

[12] X. Cabré and J. Tan, Positive solutions of nonlinear problems involving the square root of the Laplacian. Adv. Math. 224 (2010), no. 5, 2052-2093.

[13] L. Caffarelli and L. Silvestre, An extension problem related to the fractional Laplacian, Comm. Partial Differential Equations 32 (2007), no. 7-9, 1245-1260.

[14] L. A. Caffarelli, S. Salsa, and L. Silvestre, Regularity estimates for the solution and the free boundary of the obstacle problem for the fractional Laplacian, Invent. Math. 171 (2008), no. 2, 425-461.

[15] A. Capella, J. Dávila, L. Dupaigne, Y. Sire. Regularity of radial extremal solutions for some non local semilinear equations. Preprint 2010. http://www.capde.cl/publication/.

[16] J. Dávila; L. Dupaigne, Hardy-type inequalities. J. Eur. Math. Soc. (JEMS) 6 (2004), no. 3, 335-365.

[17] ] J. Dávila, L. Dupaigne, and M. Montenegro, The extremal solution of a boundary reaction problem, Commun. Pure Appl. Anal. 7 (2008), no. 4, 795-817.

[18] L. Dupaigne, Semilinear elliptic PDEs with a singular potential, J. Anal. Math., 86 (2002), 359-398.

[19] L. Dupaigne and G. Nedev. Semilinear elliptic PDEs with a singular potential. Adv. Differential Equations 7 (2002), 973-1002. 
[20] E. B. Fabes, C. E. Kenig, and R. P. Serapioni, The local regularity of solutions of degenerate elliptic equations, Comm. Partial Differential Equations 7 (1982), no. $1,77-116$.

[21] M. M. Fall, On the Hardy Poincaré inequality with boundary singularities. Commun. Contemp. Math, to appear.

[22] M. M. Fall, R. Musina, Hardy-Poincaré inequality with boundary singularities. Proc. Roy. Soc. Edinburgh, to appear.

[23] M. M. Fall, R. Musina, Sharp nonexistence results for a linear elliptic inequality involving Hardy and Leray potentials. J. Inequal. Appl. 2011 (2011). doi:10.1155/2011/917201.

[24] M. M. Fall, Nonexistence of distributional supersolutions of a semilinear elliptic equation with Hardy potential (preprint 2011).

[25] P. Felmer, A. Quaas, J. Tan, Positive solutions of Nonlinear Schrödinger equation with the fractional Laplacian. Preprint 2010.

[26] A. Fröhlich, The Helmholtz decomposition of weighted $L^{q}$-spaces for Muckenhoupt weights. Ann. Univ. Ferrara Sez. V. 46, 1 (2000), 11-19.

[27] F. Gazzola, H.-C. Grunau, E. Mitidieri, Hardy inequalities with optimal constants and remainder terms, Trans. Amer. Math. Soc. 356 (2004), 2149-2168.

[28] P. Grisvard, Elliptic problems in nonsmooth domains. Monographs and Studies in Mathematics, 24. Pitman (Advanced Publishing Program), Boston, MA, 1985.

[29] I. W. Herbst, Spectral theory of the operator $\left(p^{2}+m^{2}\right)^{1 / 2}-Z e^{2} / r$. Comm. Math. Phys. 53 (1977), no. 3, 285-294.

[30] T. Kilpeläinen, Weighted Sobolev spaces and capacity. Ann. Acad. Sci. Fenn. Ser. A I Math. 19 (1994), no. 1, 95-113.

[31] V. Kondratieva, V. Liskevich Z. Sobolb, Positive supersolutions to semi-linear second-order non-divergence type elliptic equations in exterior domains. Trans. Amer. Math. Soc. Volume 361 (2009), 697-713. 
[32] V. Kondratieva, V. Liskevich Z. Sobolb, Positive solutions to semi-linear and quasi-linear second-order elliptic equations on unbounded domains. Handbook of Differential Equation. Stationary Partial Differential Equations, volume 6 (Edited by M. Chipot) 2008 Elsevier, pp.177-268.

[33] N. S. Landkof. Foundations of modern potential theory. Springer-Verlag, New York, 1972. Translated from the Russian by A. P. Doohovskoy, Die Grundlehren der mathematischen Wissenschaften, Band 180.

[34] J. L. Lions, Théorémes de trace et d'interpolation. I. Ann. Scuola Norm. Sup. Pisa (3) 131959 389-403.

[35] V. Liskevich, S. Lyakhova and V. Moroz, Positive solutions to singular semilinear elliptic equations with critical potential on cone-like domains, Adv. Differential Equations 11 (2006), pp. 361-398.

[36] V. Liskevich, S. Lyakhova and V. Moroz, Positive solutions to semilinear elliptic equations with critical lower order terms. EQUADIFF 2003, 549-554, World Sci. Publ., Hackensack, NJ, 2005.

[37] V. Kondratieva, V. Liskevich Z. Sobolb, Second-order semilinear elliptic inequalities in exterior domains. J. of Differential Equations, vol. 187 (2003), 429455.

[38] V.G. Maz’ja, Sobolev Spaces, Springer-Verlag, 1985.

[39] S. I. Pohozaev and A. Tesei, Nonexistence of local solutions to semilinear partial differential inequalities. Ann. Inst. H. Poincaré Anal. Non Linéaire 21 (2004), pp. 487-502.

[40] R. L. Frank, A Simple Proof of Hardy-Lieb-Thirring Inequalities. Commun. Math. Phys. 290, 789-800 (2009).

[41] L. Silvestre, Regularity of the obstacle problem for a fractional power of the Laplace operator. Comm. Pure Appl. Math., 60(1):67-112, 2007.

[42] Y. Sire, E. Valdinoci, Fractional Laplacian phase transitions and boundary reactions: a geometric inequality and a symmetry result, Journal of Functional Analysis, 256, 6 (2009), 1842-1864. 
[43] E. M. Stein and G. Weiss, Fractional integrals on $n$-dimensional Euclidean space. J. Math. Mech. 71958 503-514.

[44] E. M. Stein and G. Weiss, Introduction to Fourier analysis on Euclidean spaces. Princeton Mathematical Series, No. 32. Princeton University Press, Princeton, N.J., 1971.

[45] S. Terracini, On positive entire solutions to a class of equations with a singular coefficient and critical exponent. Adv. Differential Equations 1 (1996), no. 2, 241264.

[46] J. L. Vázquez, E. Zuazua, The Hardy inequality and the asymptotic behavior of the heat equation with an inverse-square potential, J. Funct. Anal. 173 (2000).

[47] D. Yafaev, Sharp constants in the Hardy-Rellich inequalities. J. Funct. Anal. 168 (1999), no. 1, 121-144. 